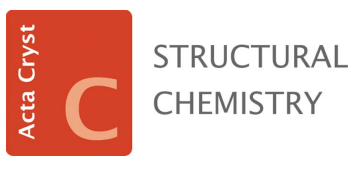

ISSN 2053-2296

Received 6 May 2016

Accepted 8 October 2016

Edited by J. White, The University of Melbourne, Australia

Keywords: pharmaceutical solvates; sulfamethazine; SMZ; benzenesulfonamide; crystal structure; triple sulfa drug.

CCDC references: 947533; 966910

Supporting information: this article has supporting information at journals.iucr.org/C

\section{Pyridine and 3-methylpyridine solvates of the triple sulfa drug constitutent sulfamethazine}

\author{
Urmila H. Patel* and Ketankumar P. Purohit
}

Department of Physics, Sardar Patel University, Vallabh Vidyanagar 388 120, Gujarat, India. *Correspondence e-mail: u_h_patel@yahoo.com

Sulfonamides display a wide variety of pharmacological activities. Sulfamethazine [abbreviated as SMZ; systematic name 4-amino- $N$-(4,6-dimethylpyrimidin2-yl)benzenesulfonamide], one of the constitutents of the triple sulfa drugs, has wide clinical use. Pharmaceutical solvates are crystalline solids of active pharmaceutical ingredients (APIs) incorporating one or more solvent molecules in the crystal lattice, and these have received special attention, as the solvent molecule can impart characteristic physicochemical properties to APIs and solvates, therefore playing a significant role in drug development. The ability of SMZ to form solvates has been investigated. Both pyridine and 3-methylpyridine form solvates with SMZ in 1:1 molar ratios. The pyridine monosolvate, $\mathrm{C}_{12} \mathrm{H}_{14} \mathrm{~N}_{4} \mathrm{O}_{2} \mathrm{~S} \cdot \mathrm{C}_{5} \mathrm{H}_{5} \mathrm{~N}$, crystallizes in the orthorhombic space group Pna $2_{1}$, with $Z=8$ and two molecules per assymetric unit, whereas the 3-methylpyridine monosolvate, $\mathrm{C}_{12} \mathrm{H}_{14} \mathrm{~N}_{4} \mathrm{O}_{2} \mathrm{~S} \cdot \mathrm{C}_{6} \mathrm{H}_{7} \mathrm{~N}$, crystallizes in the orthorhombic space group $P 2_{1} 2_{1} 2_{1}$, with $Z=4$. Crystal structure analysis reveals intramolecular $\mathrm{N}-\mathrm{H} \cdots \mathrm{N}$ hydrogen bonds between the molecules of SMZ and the pyridine solvent molecules. The solvent molecules in both structures play an active part in strong intermolecular interactions, thereby contributing significantly to the stability of both structures. Three-dimensional hydrogenbonding networks exist in both structures involving at least one sulfonyl $\mathrm{O}$ atom and the amine $\mathrm{N}$ atom. In the pyridine solvate, there is a short $\pi-\pi$ interaction [centroid-centroid distance $=3.926(3) \AA$ ] involving the centroids of the pyridine rings of two solvent molecules and a weak intermolecular $\mathrm{C}-\mathrm{H} \cdots \pi$ interaction also contributes to the stability of the crystal packing.

\section{Introduction}

Sulfonamides are recognized for their wide variety of pharmacological activities, including antibacterial, antitumor, anticarbonic anhydrase, hypoglycaemic, antithyroid and protease inhibitory activity. Clinically useful sulfonamides are derived from sulfanilamide, which is similar to 4-aminobenzoic acid, a factor required by bacteria for folic acid synthesis (Wolff, 1996). Sulfamethazine [abbreviated as SMZ; systematic name: 4-amino- $N$-(4,6-dimethylpyrimidin-2-yl)benzenesulfonamide], one of the constitutents of the triple sulfa drugs, has wide clinical use. The crystal structure of SMZ has been reported (Basak et al., 1983; Tiwari et al., 1984). Pharmaceutical solvates, crystalline solids of active pharmaceutical ingredients (APIs) which incorporate one or more solvent molecules in the crystal lattice, have received special attention as the presence of a particular solvent in the crystal lattice can impart characteristic physicochemical properties to the APIs. Therefore, solvates play a significant role in drug development (Byrn et al., 1999; Lee et al., 2011). As per our ongoing research program on crystallographic investigations of different derivatives of sulfonamides and their molecular 
Table 1

Experimental details.

Crystal data

Chemical formula

$M_{\mathrm{r}}$

Crystal system, space group

Temperature (K)

$a, b, c(\AA)$

$V\left(\AA^{3}\right)$

$Z$

Radiation type

$\mu\left(\mathrm{mm}^{-1}\right)$

Crystal size (mm)

Data collection

Diffractometer

No. of measured, independent and observed

$[I>2 \sigma(I)]$ reflections

$R_{\text {int }}$

$(\sin \theta / \lambda)_{\max }\left(\AA^{-1}\right)$

(1)

\author{
$\mathrm{C}_{12} \mathrm{H}_{14} \mathrm{~N}_{4} \mathrm{O}_{2} \mathrm{~S} \cdot \mathrm{C}_{5} \mathrm{H}_{5} \mathrm{~N}$ \\ 357.43 \\ Orthorhombic, Pna2 1 \\ 293 \\ 30.5388 (9), 8.0984 (2), 15.0810 (4) \\ $3729.76(17)$ \\ 8 \\ Mo $K \alpha$ \\ 0.19 \\ $0.65 \times 0.30 \times 0.25$
}

(2)

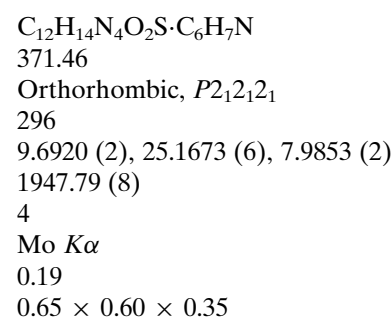

Bruker Kappa APEXII CCD 10022, 4438, 4082

0.022

0.650

$0.037,0.102,1.03$
4438
270
0
$\mathrm{H}$ atoms treated by a mixture of independent
$\quad$ and constrained refinement
$0.23,-0.28$
Refined as an inversion twin (Flack, 1983)
$-0.01(9)$

$0.047,0.105,1.00$

6825

517

$\mathrm{H}$ atoms treated by a mixture of independent and constrained refinement

$0.19,-0.19$

Refined as an inversion twin (Flack, 1983) $0.04(8)$

$\Delta \rho_{\max }, \Delta \rho_{\min }\left(\mathrm{e} \AA^{-3}\right)$

Absolute structure

Absolute structure parameter

Computer programs: APEX2 (Bruker, 2007), SAINT (Bruker, 2007), SHELXS97 (Sheldrick, 2008), SHELXL2013 (Sheldrick, 2015), SHELXL2016 (Sheldrick, 2015), PLATON (Spek, 2009), ORTEP-3 (Farrugia, 2012) and publCIF (Westrip, 2010).

solvates (Tailor et al., 2015), we report here the crystal structure of SMZ as the solvates of pyridine and 3-methylpyridine, i.e. (1) and (2), respectively.<smiles>Cc1cc(C)nc(NS(=O)(=O)c2ccc(N)cc2)n1</smiles>

(1)<smiles>Cc1cccnc1</smiles>

(2)

\section{Experimental}

\subsection{Synthesis and crystallization}

Crystals of the title sulfamethazine (SMZ) solvates were grown by slow evaporation from a saturated solution of SMZ in the respective solvent [i.e. pyridine for (1) and 3-methylpyridine for (2)]. The solutions were allowed to stand at room temperature for a few days. Tiny transparent single crystals were collected and allowed to dry in the air.

\subsection{Refinement}

Crystal data, data collection and structure refinement details are summarized in Table 1 . The $\mathrm{H}$ atoms were positioned geometrically, with $\mathrm{N}-\mathrm{H}=0.90 \AA$ for $\mathrm{NH}_{2}, \mathrm{C}-\mathrm{H}=$ $0.96 \AA$ for $\mathrm{CH}_{3}$ and $\mathrm{C}-\mathrm{H}=0.93 \AA$ for aromatic $\mathrm{H}$ atoms. In addition, the $\mathrm{H}$ atoms are constrained to ride on their parent atoms, with $U_{\text {iso }}(\mathrm{H})=1.5 U_{\text {eq }}(\mathrm{C})$ for methyl $\mathrm{H}$ atoms and $1.2 U_{\text {eq }}(\mathrm{C}, \mathrm{N})$ otherwise.

\section{Results and discussion}

The pyridine solvate of SMZ, (1), crystallizes in the orthorhombic space group $P n a 2_{1}$, with $Z=4$ and two molecules of SMZ and two molecules of pyridine in the asymmetric unit. The 3-methylpyridine solvate of SMZ, (2), crystallizes in the orthorhombic space group $P 2_{1} 2_{1} 2_{1}$, also with $Z=4$. In both solvates (Figs. 1 and 2), the bond lengths and angles of SMZ are comparable with those found in the unsolvated molecule (Basak et al., 1983; Tiwari et al., 1984). The endocyclic angles at atom $\mathrm{C} 12$ [127.3 (3) ${ }^{\circ}$ for molecule $A$ and $128.2(4)^{\circ}$ for molecule $B$ in (1), and $127.8(2)^{\circ}$ in (2)] are similar to that observed in the unsolvated molecule [129.5 (1) (Tiwari et al., 1984) and $129.1(1)^{\circ}$ (Basak et al., 1983)]. The distorted tetrahedral geometries around the $\mathrm{S}$ atom for both the solvates are also analogous to that found in the unsolvated molecule (Basak et al., 1983; Tiwari et al., 1984). The angle of inclination between the planes of the two six-membered rings of SMZ is $89.03(15)^{\circ}$ in molecule $A$ of (1), $89.40(16)^{\circ}$ in 


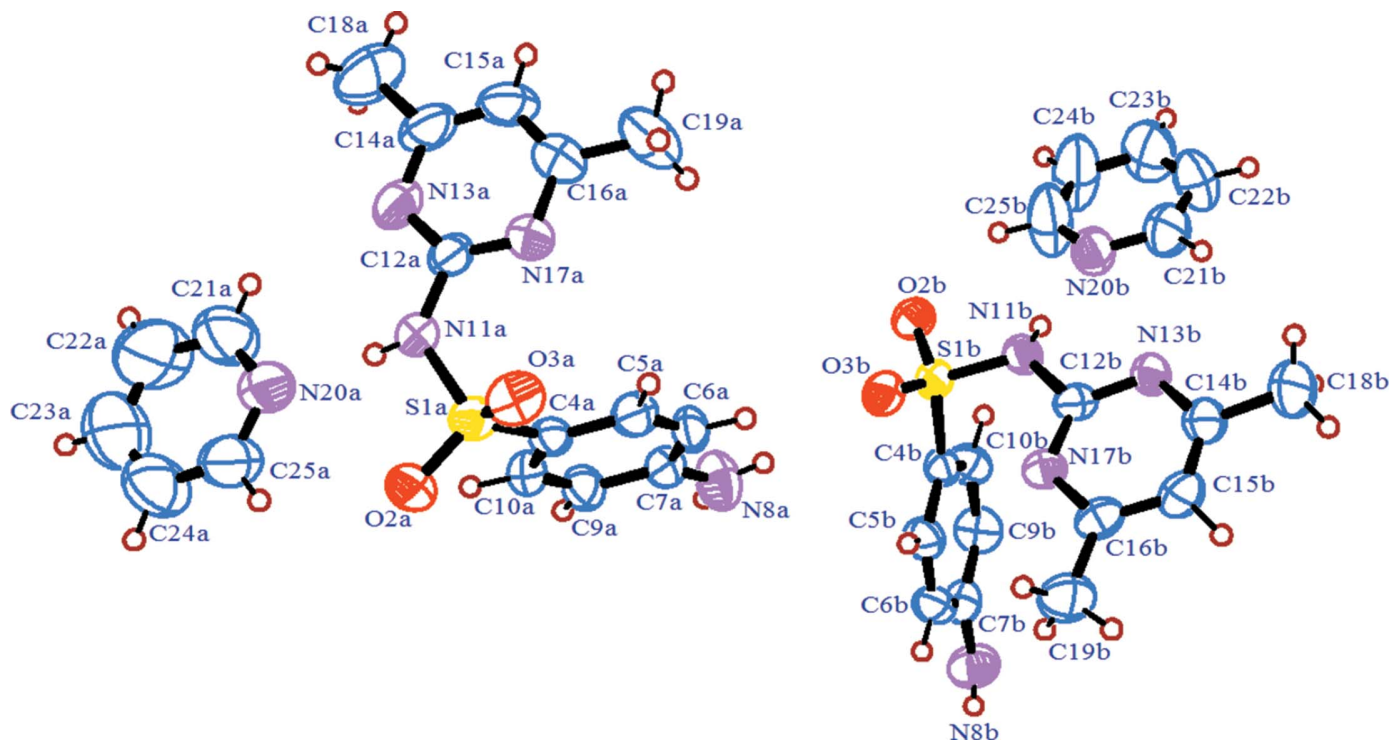

Figure 1

The molecular structure of sulfamethazine pyridine monosolvate, (1). Displacement ellipsoids are drawn at the $50 \%$ probability level.

molecule $B$ of (1) and $82.81(10)^{\circ}$ for (2), which are different than those of the unsolvated molecule [78.1 (Tiwari et al., 1984) and $75.5^{\circ}$ (Basak et al., 1983)]. The planes of the arene ring of SMZ and the pyridine ring of the solvent molecule are nearly perpendicular to each other in both solvates. The solvated structures are generally similar to each other; the orientation of the arene ring described by the $\mathrm{C} 10-\mathrm{C} 4-\mathrm{S} 1-$ N11 torsion angle [55.1 (3) ${ }^{\circ}$ in the unsolvated molecule; Basak et al., 1983] is $75.9(3)^{\circ}$ for molecule $A$ of (1), $78.1(4)^{\circ}$ for
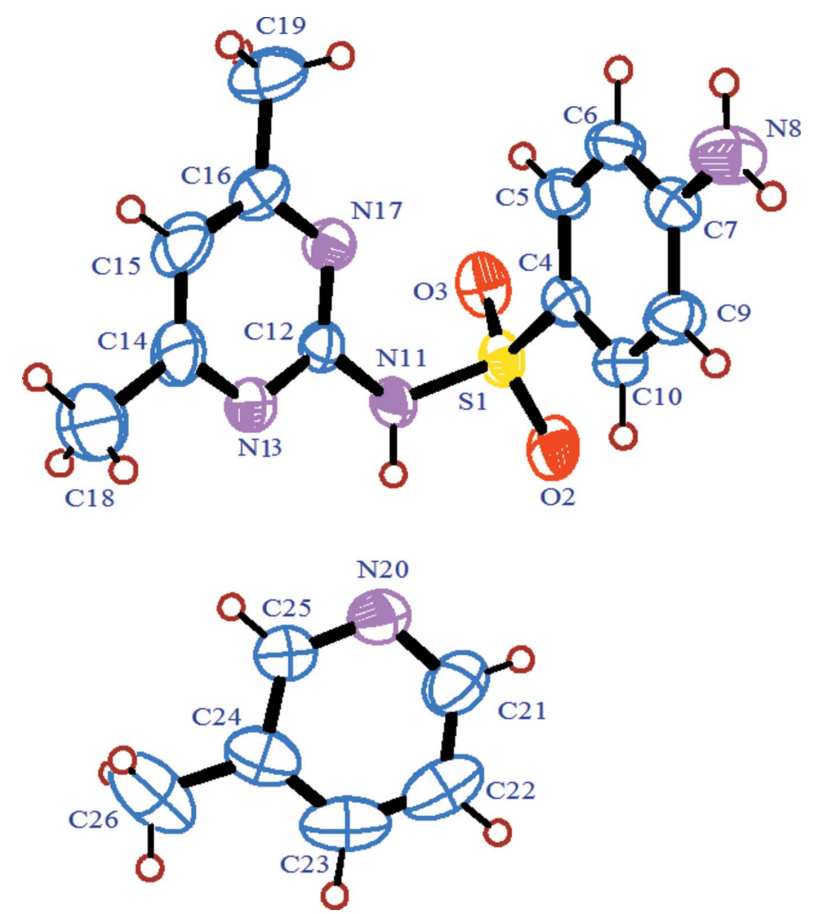

Figure 2

The molecular structure of sulfamethazine 3-methylpyridine monosolvate, (2). Displacement ellipsoids are drawn at the $50 \%$ probability level. molecule $B$ of (1) and 79.7 (2) ${ }^{\circ}$ for (2). The orientation of the pyridine ring described by the $\mathrm{N} 17-\mathrm{C} 12-\mathrm{N} 11-\mathrm{S} 1$ torsion angle is $-12.0(6)^{\circ}$ for molecule $A$ of $(1),-10.8(5)^{\circ}$ for molecule $B$ of (1) and 18.1 (3) ${ }^{\circ}$ for (2). The orientation of the molecule about the $\mathrm{S} 1-\mathrm{N} 11$ bond $\left[83.0(3)^{\circ}\right.$ in the unsolvated molecule; Basak et al., 1983] is 58.9 (4) ${ }^{\circ}$ for molecule $A$ of (1), 56.1 (4) ${ }^{\circ}$ for molecule $B$ of (1) and 49.5 (2) ${ }^{\circ}$ for (2).

In (1), the SMZ and pyridine molecules are linked via a hydrogen-bond interaction involving the sulfonyl $\mathrm{N}-\mathrm{H}$ group of SMZ and the pyridine $\mathrm{N}$ atom (Fig. 3 and Table 2). In addition, the amino $\mathrm{N}$ atom of molecule $B(\mathrm{~N} 8 B)$ hydrogen bonds via $\mathrm{H} 81 B$ to both sulfonyl atom $\mathrm{O} 3 B^{\mathrm{i}}$ and pyrimidine atom $\mathrm{N} 17 B^{\mathrm{i}}$ of an adjacent SMZ molecule, while the amino $\mathrm{N}$ atom of molecule $A$ (N8A) hydrogen bonds via atom $\mathrm{H} 82 A$ to sulfonyl atom $\mathrm{O} 3 A$ of the same SMZ molecule and via $\mathrm{H} 81 A$ to sulfonyl atom $\mathrm{O} 3 B$ of an adjacent SMZ molecule. These hydrogen bonds link the molecules along the $b$ axis. The two pyridine solvent molecules associated with molecules $A$ and $B$

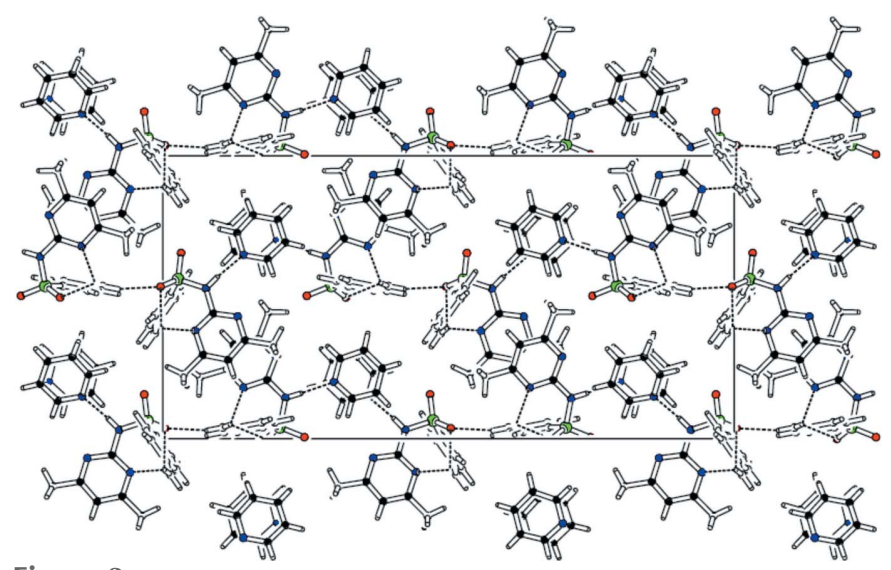

Figure 3

Packing diagram showing the hydrogen-bonding interactions in (1). The symmetry codes are as in Table 2 . 
Table 2

Hydrogen-bond geometry $\left(\AA,^{\circ}\right)$ for $(1)$.

$\mathrm{Cg} 3$ is the centroid of the $\mathrm{C} 4-\mathrm{C} 7 / \mathrm{C} 9 / \mathrm{C} 10$ ring.

\begin{tabular}{lllll}
\hline$D-\mathrm{H} \cdots A$ & $D-\mathrm{H}$ & $\mathrm{H} \cdots A$ & $D \cdots A$ & $D-\mathrm{H} \cdots A$ \\
\hline $\mathrm{N} 8 A-\mathrm{H} 81 A \cdots \mathrm{O} 3 B$ & $0.81(5)$ & $2.34(5)$ & $3.176(6)$ & $172(5)$ \\
$\mathrm{N} 8 A-\mathrm{H} 82 A \cdots \mathrm{O} 3 A$ & $0.78(4)$ & $2.49(4)$ & $3.168(6)$ & $139(4)$ \\
$\mathrm{N} 8 B-\mathrm{H} 81 B \cdots \mathrm{O} 3 B^{\mathrm{i}}$ & $0.82(5)$ & $2.59(5)$ & $3.275(6)$ & $142(4)$ \\
$\mathrm{N} 8 B-\mathrm{H} 81 B \cdots \mathrm{N} 17 B^{\mathrm{i}}$ & $0.82(5)$ & $2.55(5)$ & $3.261(6)$ & $145(4)$ \\
$\mathrm{N} 11 A-\mathrm{H} 11 A \cdots \mathrm{N} 20 A$ & $0.81(4)$ & $2.09(5)$ & $2.894(6)$ & $171(5)$ \\
$\mathrm{N} 11 B-\mathrm{H} 11 B \cdots \mathrm{N} 20 B$ & $0.80(4)$ & $2.04(4)$ & $2.834(5)$ & $174(3)$ \\
$\mathrm{C} 15 A-\mathrm{H} 15 A \cdots C g 3^{\mathrm{ii}}$ & $0.93(4)$ & 2.85 & $3.765(5)$ & 170 \\
\hline
\end{tabular}

Symmetry code: (i) $x, y-1, z$; (ii) $-x+\frac{1}{2}, y-\frac{1}{2}, z+\frac{1}{2}$.

Table 3

Hydrogen-bond geometry $\left(\AA{ }^{\circ}\right)$ for $(2)$.

\begin{tabular}{lllll}
\hline$D-\mathrm{H} \cdots A$ & $D-\mathrm{H}$ & $\mathrm{H} \cdots A$ & $D \cdots A$ & $D-\mathrm{H} \cdots A$ \\
\hline $\mathrm{N} 8-\mathrm{H} 8 A \cdots \mathrm{O} 3^{\mathrm{i}}$ & 0.87 & 2.31 & $3.1792(3)$ & 173 \\
$\mathrm{~N} 8-\mathrm{H} 8 B \cdots \mathrm{O} 3^{\text {ii }}$ & 0.88 & 2.57 & $3.2821(3)$ & 139 \\
$\mathrm{~N}^{\mathrm{H}}-\mathrm{H} 8 B \cdots \mathrm{N} 17^{\mathrm{ii}}$ & 0.88 & 2.46 & $3.1984(3)$ & 141 \\
$\mathrm{~N} 11-\mathrm{H} 11 \cdots \mathrm{N} 20$ & 0.96 & 1.90 & $2.8550(3)$ & 174 \\
\hline
\end{tabular}

Symmetry codes: (i) $x+\frac{1}{2},-y+\frac{1}{2},-z+1$; (ii) $x, y, z-1$.

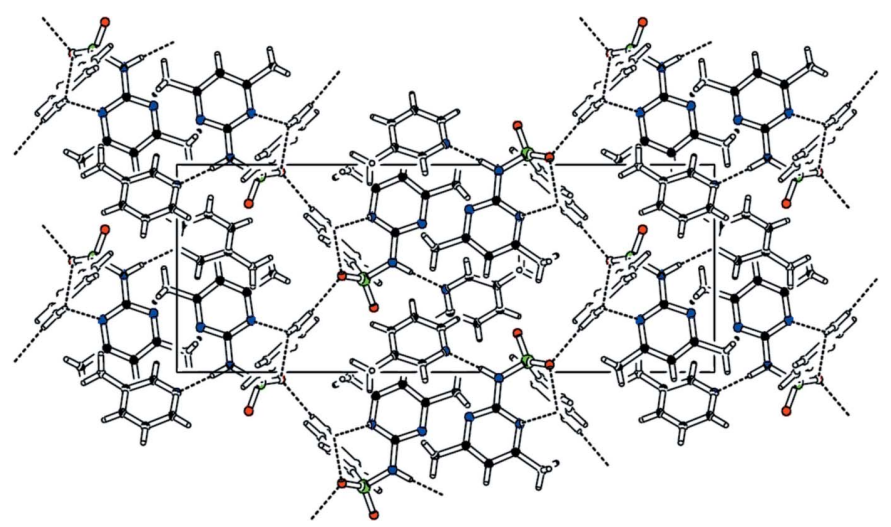

Figure 4

Packing diagram showing the hydrogen-bonding interactions (dashed lines) in (2).

are linked by a weak but significant $\pi-\pi$ interaction $\left[C g 1 \cdots C g 2\left(-x+\frac{1}{2}, y-\frac{1}{2}, z-\frac{1}{2}\right)=3.926\right.$ (3) $\AA$; $C g 1$ and $C g 2$ are the centroids of the pyridine rings $\mathrm{N} 20 A / \mathrm{C} 21 A-\mathrm{C} 25 A$ and $\mathrm{N} 20 B / \mathrm{C} 21 B-\mathrm{C} 25 B$, respectively, of the solvent molecules]; there is also a $\mathrm{C}-\mathrm{H} \cdots \pi$ interaction involving atom $\mathrm{C} 15 \mathrm{~A}$, via $\mathrm{H} 15 \mathrm{~A}$, to the centroid $\left(\mathrm{Cg} 3^{\mathrm{ii}}\right.$; Table 2$)$ of an arene ring (atoms $\mathrm{C} 4-\mathrm{C} 7 / \mathrm{C} 9 / \mathrm{C} 10)$ of an adjacent SMZ molecule.

In (2), the 3-methylpyridine solvent molecule and the pyrimidine residue of SMZ lie in the $a b$ plane and are arranged in a row along the $a$ axis, as highlighted in Fig. 4. The SMZ molecule and the 3-methylpyridine solvent molecule are linked via a hydrogen bond between the sulfonyl $\mathrm{N}-\mathrm{H}$ group of SMZ and the pyridine $\mathrm{N}$ atom (Table 3), while the amine $\mathrm{N}-\mathrm{H}$ group of SMZ is hydrogen bonded to one of the sulfonyl $\mathrm{O}$ atoms of two adjacent SMZ molecules.

\section{Acknowledgements}

We are thankful to DST, New Delhi, for providing the singlecrystal diffractometer (Kappa APEXII) at the Department of Physics, Sardar Patel University, Vallabh Vidyanagar, Gujarat, under the DST-FIST facility. KPP is also thankful to UGC for financial support (RFSMS) to carry out research work.

\section{References}

Basak, A. K., Mazumdar, S. K. \& Chaudhuri, S. (1983). Acta Cryst. C39, 492-494.

Bruker (2007). APEX2 and SAINT. Bruker AXS Inc., Madison, Wisconsion, USA.

Byrn, S. R., Pfeiffer, R. R. \& Stowell, J. G. (1999). In Solid-State Chemistry of Drugs, 2nd ed. West Lafayette, IN, USA: SSCI Inc.

Farrugia, L. J. (2012). J. Appl. Cryst. 45, 849-854.

Flack, H. D. (1983). Acta Cryst. A39, 876-881.

Lee, A. Y., Erdemir, D. \& Myerson, A. L. (2011). Annu. Rev. Chem. Biomol. Eng. 2, 259-280.

Sheldrick, G. M. (2008). Acta Cryst. A64, 112-122.

Sheldrick, G. M. (2015). Acta Cryst. C71, 3-8.

Spek, A. L. (2009). Acta Cryst. D65, 148-155.

Tailor, S. M. \& Patel, U. H. (2015). Acta Cryst. C71, 944-953.

Tiwari, R. K., Haridas, M. \& Singh, T. P. (1984). Acta Cryst. C40, 655657.

Westrip, S. P. (2010). J. Appl. Cryst. 43, 920-925.

Wolff, M. E. (1996). Burger's Medicinal Chemistry and Drug Discovery, Vol. 2, 5th ed., pp. 528-576. New York: John Wiley \& Sons Inc. 


\section{supporting information}

Acta Cryst. (2017). C73, 9-12 [https://doi.org/10.1107/S2053229616015898]

\section{Pyridine and 3-methylpyridine solvates of the triple sulfa drug constitutent sulfamethazine}

\section{Urmila H. Patel and Ketankumar P. Purohit}

Computing details

For both compounds, data collection: APEX2 (Bruker, 2007); cell refinement: SAINT (Bruker, 2007); data reduction: SAINT (Bruker, 2007); program(s) used to solve structure: SHELXS97 (Sheldrick, 2008). Program(s) used to refine structure: SHELXL2013 (Sheldrick, 2015) for (1); SHELXL2016 (Sheldrick, 2015) for (2). For both compounds, molecular graphics: PLATON (Spek, 2009) and ORTEP-3 (Farrugia, 2012); software used to prepare material for publication: publCIF (Westrip, 2010).

(1) 4-Amino- $N$-(4,6-dimethylpyrimidin-2-yl)benzenesulfonamide pyridine monosolvate

Crystal data

$\mathrm{C}_{12} \mathrm{H}_{14} \mathrm{~N}_{4} \mathrm{O}_{2} \mathrm{~S} \cdot \mathrm{C}_{5} \mathrm{H}_{5} \mathrm{~N}$

$M_{r}=357.43$

Orthorhombic, Pna2 1

$a=30.5388(9) \AA$

$b=8.0984(2) \AA$

$c=15.0810(4) \AA$

$V=3729.76(17) \AA^{3}$

$Z=8$

$F(000)=1504$

\section{Data collection}

Bruker Kappa APEXII CCD diffractometer

$\varphi$ and $\omega$ scans

18564 measured reflections

6825 independent reflections

4494 reflections with $I>2 \sigma(I)$

\section{Refinement}

Refinement on $F^{2}$

Least-squares matrix: full

$R\left[F^{2}>2 \sigma\left(F^{2}\right)\right]=0.047$

$w R\left(F^{2}\right)=0.105$

$S=1.00$

6825 reflections

517 parameters

1 restraint

Hydrogen site location: mixed
$D_{\mathrm{x}}=1.273 \mathrm{Mg} \mathrm{m}^{-3}$

Mo $K \alpha$ radiation, $\lambda=0.71073 \AA$

Cell parameters from 4378 reflections

$\theta=2.7-21.2^{\circ}$

$\mu=0.19 \mathrm{~mm}^{-1}$

$T=293 \mathrm{~K}$

Needle, colourless

$0.65 \times 0.30 \times 0.25 \mathrm{~mm}$

$R_{\text {int }}=0.035$

$\theta_{\text {max }}=27.4^{\circ}, \theta_{\text {min }}=1.3^{\circ}$

$h=-26 \rightarrow 39$

$k=-10 \rightarrow 8$

$l=-19 \rightarrow 11$

$\mathrm{H}$ atoms treated by a mixture of independent and constrained refinement

$w=1 /\left[\sigma^{2}\left(F_{0}^{2}\right)+(0.0506 P)^{2}\right]$ where $P=\left(F_{\mathrm{o}}{ }^{2}+2 F_{\mathrm{c}}{ }^{2}\right) / 3$

$(\Delta / \sigma)_{\max }=0.005$

$\Delta \rho_{\max }=0.19 \mathrm{e} \AA^{-3}$

$\Delta \rho_{\min }=-0.19$ e $\AA^{-3}$

Extinction correction: SHELXL2013

(Sheldrick, 2015),

$\mathrm{Fc}^{*}=\mathrm{kFc}\left[1+0.001 \mathrm{xFc}^{2} \lambda^{3} / \sin (2 \theta)\right]^{-1 / 4}$ 
Extinction coefficient: 0.0016 (4)
Absolute structure: Refined as an inversion twin (Flack, 1983)

Absolute structure parameter: $0.04(8)$

Special details

Geometry. All esds (except the esd in the dihedral angle between two 1.s. planes) are estimated using the full covariance matrix. The cell esds are taken into account individually in the estimation of esds in distances, angles and torsion angles; correlations between esds in cell parameters are only used when they are defined by crystal symmetry. An approximate (isotropic) treatment of cell esds is used for estimating esds involving l.s. planes.

Refinement. Refined as a 2-component inversion twin.

Fractional atomic coordinates and isotropic or equivalent isotropic displacement parameters $\left(\AA^{2}\right)$

\begin{tabular}{|c|c|c|c|c|}
\hline & $x$ & $y$ & $z$ & $U_{\text {iso }} * / U_{\text {eq }}$ \\
\hline N20A & $-0.30004(15)$ & $0.8913(5)$ & $0.2996(3)$ & $0.0820(13)$ \\
\hline $\mathrm{C} 25 \mathrm{~A}$ & $-0.3316(2)$ & $0.8679(7)$ & $0.3576(4)$ & $0.0906(17)$ \\
\hline $\mathrm{H} 25 \mathrm{~A}$ & -0.324254 & 0.873117 & 0.417375 & $0.109 *$ \\
\hline $\mathrm{C} 24 \mathrm{~A}$ & $-0.3736(2)$ & $0.8368(9)$ & $0.3368(6)$ & $0.115(2)$ \\
\hline $\mathrm{H} 24 \mathrm{~A}$ & -0.394463 & 0.819105 & 0.380762 & $0.138 *$ \\
\hline $\mathrm{C} 21 \mathrm{~A}$ & $-0.3108(2)$ & $0.8853(10)$ & $0.2166(5)$ & $0.123(2)$ \\
\hline $\mathrm{H} 21 \mathrm{~A}$ & -0.289126 & 0.904116 & 0.174472 & $0.147 *$ \\
\hline $\mathrm{C} 22 \mathrm{~A}$ & -0.3520 & $0.8528(13)$ & $0.1880(6)$ & $0.167(4)$ \\
\hline $\mathrm{H} 22 \mathrm{~A}$ & -0.358288 & 0.844580 & 0.127862 & $0.200 *$ \\
\hline $\mathrm{C} 23 \mathrm{~A}$ & -0.3845 & $0.8322(11)$ & $0.2516(9)$ & $0.158(3)$ \\
\hline $\mathrm{H} 23 \mathrm{~A}$ & -0.413451 & 0.815444 & 0.234656 & $0.189 *$ \\
\hline S1B & $0.02690(3)$ & $0.27065(11)$ & $0.43631(7)$ & $0.0441(3)$ \\
\hline S1A & $-0.20516(3)$ & $0.95563(13)$ & $0.46009(7)$ & $0.0536(3)$ \\
\hline $\mathrm{O} 3 \mathrm{~B}$ & $-0.00381(8)$ & $0.3942(3)$ & $0.46281(19)$ & $0.0526(7)$ \\
\hline N17B & $0.05700(11)$ & $0.3537(4)$ & $0.6183(2)$ & $0.0497(8)$ \\
\hline $\mathrm{O} 2 \mathrm{~B}$ & $0.03220(9)$ & $0.2389(3)$ & $0.34374(18)$ & $0.0573(8)$ \\
\hline $\mathrm{C} 10 \mathrm{~B}$ & $0.02973(14)$ & $-0.0611(5)$ & $0.4606(3)$ & $0.0533(11)$ \\
\hline C7A & $-0.12822(12)$ & $0.4776(5)$ & $0.4602(3)$ & $0.0489(9)$ \\
\hline $\mathrm{C} 4 \mathrm{~A}$ & $-0.17513(12)$ & $0.7738(4)$ & $0.4598(3)$ & $0.0442(9)$ \\
\hline $\mathrm{C} 12 \mathrm{~B}$ & $0.08850(13)$ & $0.3473(4)$ & $0.5573(3)$ & $0.0436(9)$ \\
\hline N11B & $0.07562(11)$ & $0.3225(4)$ & $0.4690(2)$ & $0.0481(8)$ \\
\hline $\mathrm{O} 3 \mathrm{~A}$ & $-0.17959(11)$ & $1.0823(3)$ & $0.5007(2)$ & $0.0653(8)$ \\
\hline $\mathrm{C} 10 \mathrm{~A}$ & $-0.19598(14)$ & $0.6254(5)$ & $0.4419(3)$ & $0.0532(11)$ \\
\hline N17A & $-0.14181(12)$ & $1.0502(4)$ & $0.3192(2)$ & $0.0578(9)$ \\
\hline C6A & $-0.10755(14)$ & $0.6291(5)$ & $0.4763(3)$ & $0.0512(11)$ \\
\hline N8A & $-0.10533(17)$ & $0.3354(6)$ & $0.4608(4)$ & $0.0746(13)$ \\
\hline N13B & $0.13131(11)$ & $0.3637(4)$ & $0.5687(2)$ & $0.0541(9)$ \\
\hline $\mathrm{C} 5 \mathrm{~A}$ & $-0.13036(14)$ & $0.7736(5)$ & $0.4765(3)$ & $0.0513(11)$ \\
\hline N11A & $-0.21463(13)$ & $1.0049(5)$ & $0.3560(3)$ & $0.0583(10)$ \\
\hline $\mathrm{C} 4 \mathrm{~B}$ & $0.01261(12)$ & $0.0878(4)$ & $0.4906(3)$ & $0.0400(9)$ \\
\hline C6B & $-0.02321(14)$ & $-0.0545(5)$ & 0.6085 & $0.0530(11)$ \\
\hline $\mathrm{O} 2 \mathrm{~A}$ & $-0.24790(9)$ & $0.9224(3)$ & $0.4943(2)$ & $0.0667(9)$ \\
\hline N13A & $-0.19812(13)$ & $1.0397(5)$ & $0.2096(3)$ & $0.0705(11)$ \\
\hline C16B & $0.07060(15)$ & $0.3713(5)$ & $0.7036(3)$ & $0.0518(11)$ \\
\hline C15B & $0.11458(17)$ & $0.3878(6)$ & $0.7215(3)$ & $0.0639(12)$ \\
\hline
\end{tabular}




\begin{tabular}{|c|c|c|c|c|}
\hline $\mathrm{C} 5 \mathrm{~B}$ & $-0.01422(13)$ & $0.0901(5)$ & $0.5644(3)$ & $0.0512(10)$ \\
\hline $\mathrm{C} 12 \mathrm{~A}$ & $-0.18292(14)$ & $1.0333(5)$ & $0.2928(3)$ & $0.0503(10)$ \\
\hline C9B & $0.02067(15)$ & $-0.2048(6)$ & $0.5052(3)$ & $0.0600(12)$ \\
\hline N8B & $-0.01412(18)$ & $-0.3465(6)$ & $0.6281(4)$ & $0.0688(13)$ \\
\hline $\mathrm{C} 15 \mathrm{~A}$ & $-0.1248(2)$ & $1.0851(7)$ & $0.1674(4)$ & $0.0804(17)$ \\
\hline $\mathrm{C} 16 \mathrm{~A}$ & $-0.11190(16)$ & $1.0772(5)$ & $0.2545(4)$ & $0.0656(13)$ \\
\hline $\mathrm{C} 7 \mathrm{~B}$ & $-0.00593(13)$ & $-0.2046(5)$ & $0.5817(3)$ & $0.0525(11)$ \\
\hline C19B & $0.03641(17)$ & $0.3704(7)$ & $0.7725(3)$ & $0.0792(15)$ \\
\hline H191 & 0.049730 & 0.383666 & 0.829738 & $0.119^{*}$ \\
\hline H192 & 0.016349 & 0.459635 & 0.762120 & $0.119^{*}$ \\
\hline H193 & 0.020867 & 0.267458 & 0.770616 & $0.119^{*}$ \\
\hline C9A & $-0.17331(13)$ & $0.4795(5)$ & $0.4425(3)$ & $0.0549(10)$ \\
\hline C14B & $0.14401(15)$ & $0.3858(6)$ & $0.6528(3)$ & $0.0596(12)$ \\
\hline C18B & $0.19249(16)$ & $0.4075(8)$ & $0.6669(4)$ & $0.0966(19)$ \\
\hline H183 & 0.198278 & 0.422347 & 0.728971 & $0.145^{*}$ \\
\hline H182 & 0.207671 & 0.311243 & 0.646000 & $0.145^{*}$ \\
\hline H181 & 0.202439 & 0.502674 & 0.634770 & $0.145^{*}$ \\
\hline $\mathrm{C} 14 \mathrm{~A}$ & $-0.1681(2)$ & $1.0682(6)$ & $0.1464(3)$ & $0.0781(16)$ \\
\hline C19A & $-0.06528(16)$ & $1.0934(7)$ & $0.2830(4)$ & $0.0945(18)$ \\
\hline H19X & -0.047145 & 1.112204 & 0.231963 & $0.142 *$ \\
\hline H19Z & -0.062509 & 1.184781 & 0.323170 & $0.142 *$ \\
\hline H19Y & -0.056193 & 0.993779 & 0.312124 & $0.142 *$ \\
\hline C18A & $-0.1855(2)$ & $1.0738(10)$ & $0.0532(4)$ & $0.135(3)$ \\
\hline $\mathrm{H} 18 \mathrm{Z}$ & -0.161873 & 1.094631 & 0.012719 & $0.202^{*}$ \\
\hline $\mathrm{H} 18 \mathrm{X}$ & -0.198941 & 0.969936 & 0.038944 & $0.202^{*}$ \\
\hline H18Y & -0.206839 & 1.160412 & 0.048313 & $0.202 *$ \\
\hline N20B & $0.14115(12)$ & $0.2099(5)$ & $0.3505(3)$ & $0.0682(10)$ \\
\hline $\mathrm{C} 21 \mathrm{~B}$ & $0.18363(18)$ & $0.2129(6)$ & $0.3658(4)$ & $0.0786(15)$ \\
\hline $\mathrm{H} 21 \mathrm{~B}$ & 0.193231 & 0.251730 & 0.420524 & $0.094^{*}$ \\
\hline $\mathrm{C} 25 \mathrm{~B}$ & $0.1298(2)$ & $0.1555(10)$ & $0.2723(4)$ & $0.125(3)$ \\
\hline $\mathrm{H} 25 \mathrm{~B}$ & 0.100148 & 0.155104 & 0.258292 & $0.150^{*}$ \\
\hline $\mathrm{C} 23 \mathrm{~B}$ & $0.2015(2)$ & $0.1057(9)$ & $0.2257(5)$ & $0.110(2)$ \\
\hline $\mathrm{H} 23 \mathrm{~B}$ & 0.221605 & 0.072602 & 0.183023 & $0.132 *$ \\
\hline $\mathrm{C} 24 \mathrm{~B}$ & $0.1583(2)$ & $0.0993(11)$ & 0.2099 (4) & $0.140(3)$ \\
\hline H24B & 0.147917 & 0.056723 & 0.156685 & $0.168^{*}$ \\
\hline $\mathrm{C} 22 \mathrm{~B}$ & $0.21464(17)$ & $0.1617(7)$ & $0.3053(5)$ & $0.0904(17)$ \\
\hline H22B & 0.244274 & 0.165852 & 0.319309 & $0.108^{*}$ \\
\hline H9A & $-0.1899(12)$ & $0.378(5)$ & $0.427(3)$ & $0.059(12)^{*}$ \\
\hline H82A & $-0.1168(14)$ & $0.252(5)$ & $0.449(3)$ & $0.058(16)^{*}$ \\
\hline $\mathrm{H} 5 \mathrm{~A}$ & $-0.1172(12)$ & $0.881(5)$ & $0.484(2)$ & $0.049(11)^{*}$ \\
\hline H10B & $0.0500(11)$ & $-0.054(4)$ & $0.411(3)$ & $0.046(10)^{*}$ \\
\hline H5B & $-0.0245(12)$ & $0.199(5)$ & $0.591(3)$ & $0.061(12)^{*}$ \\
\hline $\mathrm{H} 10 \mathrm{~A}$ & $-0.2265(13)$ & $0.628(4)$ & $0.429(3)$ & $0.055(11)^{*}$ \\
\hline H15B & $0.1231(12)$ & $0.389(4)$ & $0.784(3)$ & $0.049(11)^{*}$ \\
\hline H9B & $0.0287(13)$ & $-0.307(5)$ & $0.484(3)$ & $0.066(14)^{*}$ \\
\hline H81A & $-0.0789(16)$ & $0.342(5)$ & $0.465(4)$ & $0.071(16)^{*}$ \\
\hline H6A & $-0.0789(13)$ & $0.625(4)$ & 0.490 & $0.056(12)^{*}$ \\
\hline H6B & $-0.0404(14)$ & $-0.059(5)$ & $0.654(3)$ & $0.068(15)^{*}$ \\
\hline
\end{tabular}




\begin{tabular}{lllll} 
H11B & $0.0926(12)$ & $0.286(4)$ & $0.434(3)$ & $0.047(12)^{*}$ \\
H11A & $-0.2393(15)$ & $0.974(5)$ & $0.346(3)$ & $0.067(16)^{*}$ \\
H81B & $-0.0035(14)$ & $-0.430(6)$ & $0.606(3)$ & $0.064(16)^{*}$ \\
H15A & $-0.1058(19)$ & $1.108(7)$ & $0.127(5)$ & $0.11(2)^{*}$ \\
H82B & $-0.025(2)$ & $-0.347(8)$ & $0.674(5)$ & $0.10(3)^{*}$ \\
\hline
\end{tabular}

Atomic displacement parameters $\left(\AA^{2}\right)$

\begin{tabular}{|c|c|c|c|c|c|c|}
\hline & $U^{11}$ & $U^{22}$ & $U^{33}$ & $U^{12}$ & $U^{13}$ & $U^{23}$ \\
\hline N20A & $0.078(3)$ & $0.100(3)$ & $0.068(3)$ & $-0.010(2)$ & $-0.003(3)$ & $0.015(2)$ \\
\hline $\mathrm{C} 25 \mathrm{~A}$ & $0.093(4)$ & $0.107(4)$ & $0.072(4)$ & $-0.027(3)$ & $-0.004(4)$ & $0.012(3)$ \\
\hline $\mathrm{C} 24 \mathrm{~A}$ & $0.086(5)$ & $0.145(6)$ & $0.113(6)$ & $-0.036(4)$ & $0.002(5)$ & $0.000(5)$ \\
\hline $\mathrm{C} 21 \mathrm{~A}$ & $0.091(5)$ & $0.204(8)$ & $0.073(5)$ & $-0.020(5)$ & $0.002(4)$ & $0.009(5)$ \\
\hline $\mathrm{C} 22 \mathrm{~A}$ & $0.143(8)$ & $0.276(12)$ & $0.082(6)$ & $-0.057(8)$ & -0.039 (6) & $0.017(6)$ \\
\hline $\mathrm{C} 23 \mathrm{~A}$ & $0.092(6)$ & $0.215(9)$ & $0.165(10)$ & $-0.041(6)$ & $-0.032(7)$ & $0.015(8)$ \\
\hline S1B & $0.0428(5)$ & $0.0524(6)$ & $0.0373(5)$ & -0.0013 & $-0.0031(5)$ & $0.0002(5)$ \\
\hline S1A & $0.0545(6)$ & $0.0593(6)$ & $0.0471(7)$ & $0.0040(5)$ & $0.0064(6)$ & $0.0056(5)$ \\
\hline O3B & $0.0523(15)$ & $0.0511(14)$ & $0.0544(18)$ & $0.0066(12)$ & $-0.0028(15)$ & $0.0054(13)$ \\
\hline N17B & $0.051(2)$ & $0.060(2)$ & $0.038(2)$ & $-0.0011(17)$ & $0.0039(18)$ & $0.0018(16)$ \\
\hline $\mathrm{O} 2 \mathrm{~B}$ & $0.0572(17)$ & 0.0802 (19) & $0.0344(16)$ & $-0.0083(14)$ & $-0.0048(14)$ & $-0.0008(14)$ \\
\hline $\mathrm{C} 10 \mathrm{~B}$ & $0.056(2)$ & $0.057(3)$ & $0.048(3)$ & $-0.002(2)$ & $0.010(3)$ & $-0.009(2)$ \\
\hline C7A & $0.045(2)$ & $0.055(2)$ & $0.047(2)$ & $0.004(2)$ & $0.002(2)$ & $0.004(2)$ \\
\hline $\mathrm{C} 4 \mathrm{~A}$ & $0.044(2)$ & $0.055(2)$ & $0.034(2)$ & $-0.0043(18)$ & 0.0018 (19) & $0.002(2)$ \\
\hline C12B & $0.053(3)$ & $0.041(2)$ & $0.037(2)$ & $-0.0071(18)$ & $0.002(2)$ & $0.0013(17)$ \\
\hline N11B & 0.0394 (19) & $0.072(2)$ & 0.0333 (19) & $-0.0053(16)$ & $0.0031(18)$ & $-0.0053(17)$ \\
\hline $\mathrm{O} 3 \mathrm{~A}$ & $0.081(2)$ & $0.0565(17)$ & $0.058(2)$ & $0.0004(16)$ & $0.0001(17)$ & $-0.0046(13)$ \\
\hline $\mathrm{C} 10 \mathrm{~A}$ & $0.041(2)$ & $0.063(3)$ & $0.056(3)$ & $-0.002(2)$ & $-0.006(2)$ & $0.008(2)$ \\
\hline N17A & $0.052(2)$ & $0.069(2)$ & $0.052(2)$ & $-0.0009(18)$ & $0.005(2)$ & $0.0056(17)$ \\
\hline C6A & $0.034(2)$ & $0.072(3)$ & $0.048(3)$ & $0.000(2)$ & $-0.005(2)$ & $-0.001(2)$ \\
\hline N8A & $0.055(3)$ & $0.060(3)$ & $0.109(4)$ & $0.005(2)$ & $-0.004(3)$ & -0.005 \\
\hline N13B & $0.043(2)$ & $0.079(2)$ & $0.040(2)$ & $-0.0167(17)$ & $-0.0072(17)$ & $0.0001(17)$ \\
\hline C5A & $0.055(3)$ & $0.051(3)$ & $0.048(3)$ & $-0.010(2)$ & $-0.005(2)$ & 0.0015 (19) \\
\hline N11A & $0.046(2)$ & $0.076(3)$ & $0.053(3)$ & $0.003(2)$ & $0.000(2)$ & 0.0191 (19) \\
\hline C4B & $0.0341(18)$ & $0.044(2)$ & $0.042(2)$ & $0.0022(17)$ & 0.0003 (18) & $-0.0006(16)$ \\
\hline C6B & $0.048(3)$ & $0.061(3)$ & $0.051(3)$ & $0.004(2)$ & $0.012(2)$ & $0.006(2)$ \\
\hline $\mathrm{O} 2 \mathrm{~A}$ & $0.0549(17)$ & 0.0802 (19) & $0.065(2)$ & $0.0100(15)$ & $0.0162(16)$ & $0.0102(15)$ \\
\hline N13A & $0.074(3)$ & $0.083(3)$ & $0.055(3)$ & $0.015(2)$ & $-0.004(2)$ & $0.017(2)$ \\
\hline $\mathrm{C} 16 \mathrm{~B}$ & $0.066(3)$ & $0.055(2)$ & $0.034(2)$ & $0.002(2)$ & $0.001(2)$ & $-0.0030(18)$ \\
\hline C15B & $0.068(3)$ & $0.085(3)$ & $0.039(3)$ & -0.010 & $-0.011(3)$ & $0.000(2)$ \\
\hline C5B & $0.047(2)$ & $0.052(3)$ & $0.055(3)$ & $0.007(2)$ & 0.007 (2) & $0.000(2)$ \\
\hline $\mathrm{C} 12 \mathrm{~A}$ & $0.056(3)$ & $0.051(2)$ & $0.044(3)$ & $0.007(2)$ & $0.000(2)$ & 0.009 (2) \\
\hline C9B & $0.070(3)$ & $0.046(3)$ & $0.064(3)$ & $0.005(2)$ & $0.005(3)$ & $-0.011(2)$ \\
\hline N8B & $0.086(3)$ & $0.049(3)$ & $0.071(3)$ & $0.002(2)$ & $0.005(3)$ & $0.012(3)$ \\
\hline C15A & $0.089(5)$ & $0.097(4)$ & $0.055(4)$ & $-0.001(3)$ & $0.024(4)$ & $0.017(3)$ \\
\hline C16A & $0.061(3)$ & $0.067(3)$ & $0.069(4)$ & $-0.004(2)$ & $0.014(3)$ & $-0.003(2)$ \\
\hline $\mathrm{C} 7 \mathrm{~B}$ & $0.049(2)$ & $0.055(3)$ & $0.053(3)$ & $0.000(2)$ & $-0.010(2)$ & $-0.001(2)$ \\
\hline C19B & $0.084(4)$ & $0.105(4)$ & $0.049(3)$ & $0.009(3)$ & $0.012(3)$ & $-0.002(3)$ \\
\hline C9A & $0.046(2)$ & $0.053(3)$ & $0.065(3)$ & $-0.005(2)$ & $-0.001(2)$ & $0.004(2)$ \\
\hline
\end{tabular}




$\begin{array}{lllllll} & & & & & \\ \mathrm{C} 14 \mathrm{~B} & 0.055(3) & 0.074(3) & 0.050(3) & -0.018(2) & -0.008(3) & 0.002(2) \\ \mathrm{C} 18 \mathrm{~B} & 0.071(4) & 0.149(5) & 0.070(4) & -0.033(3) & -0.021(3) & 0.002(3) \\ \mathrm{C} 14 \mathrm{~A} & 0.099(4) & 0.090(4) & 0.045(3) & 0.019(3) & 0.007(3) & 0.016(3) \\ \mathrm{C} 19 \mathrm{~A} & 0.063(4) & 0.126(5) & 0.094(4) & -0.023(3) & 0.019(3) & -0.017(3) \\ \mathrm{C} 18 \mathrm{~A} & 0.152(6) & 0.196(7) & 0.056(4) & 0.017(5) & -0.008(5) & 0.035(4) \\ \mathrm{N} 20 \mathrm{~B} & 0.054(2) & 0.105(3) & 0.046(2) & -0.003(2) & -0.001(2) & -0.009(2) \\ \mathrm{C} 21 \mathrm{~B} & 0.073(4) & 0.095(4) & 0.068(4) & -0.008(3) & -0.012(3) & -0.005(3) \\ \mathrm{C} 25 \mathrm{~B} & 0.063(4) & 0.250(8) & 0.062(4) & 0.030(5) & -0.007(3) & -0.053(5) \\ \mathrm{C} 23 \mathrm{~B} & 0.076(5) & 0.167(6) & 0.086(5) & 0.034(4) & 0.021(4) & -0.017(4) \\ \mathrm{C} 24 \mathrm{~B} & 0.078(5) & 0.284(10) & 0.057(4) & 0.055(5) & -0.006(4) & -0.054(5) \\ \mathrm{C} 22 \mathrm{~B} & 0.050(3) & 0.109(4) & 0.113(6) & 0.007(3) & 0.002(4) & -0.015(4) \\ & & & & & & \end{array}$

Geometric parameters $\left(\AA,{ }^{\circ}\right)$

\begin{tabular}{|c|c|c|c|}
\hline $\mathrm{N} 20 \mathrm{~A}-\mathrm{C} 21 \mathrm{~A}$ & $1.294(8)$ & $\mathrm{C} 6 \mathrm{~B}-\mathrm{C} 5 \mathrm{~B}$ & $1.374(6)$ \\
\hline $\mathrm{N} 20 \mathrm{~A}-\mathrm{C} 25 \mathrm{~A}$ & $1.314(7)$ & $\mathrm{C} 6 \mathrm{~B}-\mathrm{C} 7 \mathrm{~B}$ & $1.385(6)$ \\
\hline $\mathrm{C} 25 \mathrm{~A}-\mathrm{C} 24 \mathrm{~A}$ & $1.347(8)$ & $\mathrm{C} 6 \mathrm{~B}-\mathrm{H} 6 \mathrm{~B}$ & $0.87(4)$ \\
\hline $\mathrm{C} 25 \mathrm{~A}-\mathrm{H} 25 \mathrm{~A}$ & 0.9300 & $\mathrm{~N} 13 \mathrm{~A}-\mathrm{C} 12 \mathrm{~A}$ & $1.339(5)$ \\
\hline $\mathrm{C} 24 \mathrm{~A}-\mathrm{C} 23 \mathrm{~A}$ & $1.327(11)$ & $\mathrm{N} 13 \mathrm{~A}-\mathrm{C} 14 \mathrm{~A}$ & $1.342(6)$ \\
\hline $\mathrm{C} 24 \mathrm{~A}-\mathrm{H} 24 \mathrm{~A}$ & 0.9300 & $\mathrm{C} 16 \mathrm{~B}-\mathrm{C} 15 \mathrm{~B}$ & $1.377(6)$ \\
\hline $\mathrm{C} 21 \mathrm{~A}-\mathrm{C} 22 \mathrm{~A}$ & $1.356(10)$ & $\mathrm{C} 16 \mathrm{~B}-\mathrm{C} 19 \mathrm{~B}$ & $1.473(6)$ \\
\hline $\mathrm{C} 21 \mathrm{~A}-\mathrm{H} 21 \mathrm{~A}$ & 0.9300 & $\mathrm{C} 15 \mathrm{~B}-\mathrm{C} 14 \mathrm{~B}$ & $1.372(6)$ \\
\hline $\mathrm{C} 22 \mathrm{~A}-\mathrm{C} 23 \mathrm{~A}$ & $1.390(12)$ & $\mathrm{C} 15 \mathrm{~B}-\mathrm{H} 15 \mathrm{~B}$ & $0.98(4)$ \\
\hline $\mathrm{C} 22 \mathrm{~A}-\mathrm{H} 22 \mathrm{~A}$ & 0.9300 & $\mathrm{C} 5 \mathrm{~B}-\mathrm{H} 5 \mathrm{~B}$ & $1.02(4)$ \\
\hline $\mathrm{C} 23 \mathrm{~A}-\mathrm{H} 23 \mathrm{~A}$ & 0.9300 & $\mathrm{C} 9 \mathrm{~B}-\mathrm{C} 7 \mathrm{~B}$ & $1.412(6)$ \\
\hline $\mathrm{S} 1 \mathrm{~B}-\mathrm{O} 3 \mathrm{~B}$ & $1.428(3)$ & $\mathrm{C} 9 \mathrm{~B}-\mathrm{H} 9 \mathrm{~B}$ & $0.92(4)$ \\
\hline $\mathrm{S} 1 \mathrm{~B}-\mathrm{O} 2 \mathrm{~B}$ & $1.429(3)$ & $\mathrm{N} 8 \mathrm{~B}-\mathrm{C} 7 \mathrm{~B}$ & $1.368(6)$ \\
\hline $\mathrm{S} 1 \mathrm{~B}-\mathrm{N} 11 \mathrm{~B}$ & $1.623(3)$ & $\mathrm{N} 8 \mathrm{~B}-\mathrm{H} 81 \mathrm{~B}$ & $0.82(4)$ \\
\hline $\mathrm{S} 1 \mathrm{~B}-\mathrm{C} 4 \mathrm{~B}$ & $1.747(4)$ & $\mathrm{N} 8 \mathrm{~B}-\mathrm{H} 82 \mathrm{~B}$ & $0.76(6)$ \\
\hline $\mathrm{S} 1 \mathrm{~A}-\mathrm{O} 3 \mathrm{~A}$ & $1.427(3)$ & $\mathrm{C} 15 \mathrm{~A}-\mathrm{C} 14 \mathrm{~A}$ & $1.367(8)$ \\
\hline $\mathrm{S} 1 \mathrm{~A}-\mathrm{O} 2 \mathrm{~A}$ & $1.429(3)$ & $\mathrm{C} 15 \mathrm{~A}-\mathrm{C} 16 \mathrm{~A}$ & $1.373(8)$ \\
\hline $\mathrm{S} 1 \mathrm{~A}-\mathrm{N} 11 \mathrm{~A}$ & $1.645(4)$ & $\mathrm{C} 15 \mathrm{~A}-\mathrm{H} 15 \mathrm{~A}$ & $0.86(6)$ \\
\hline $\mathrm{S} 1 \mathrm{~A}-\mathrm{C} 4 \mathrm{~A}$ & $1.735(4)$ & $\mathrm{C} 16 \mathrm{~A}-\mathrm{C} 19 \mathrm{~A}$ & $1.493(7)$ \\
\hline $\mathrm{N} 17 \mathrm{~B}-\mathrm{C} 12 \mathrm{~B}$ & $1.332(5)$ & C19B-H191 & 0.9600 \\
\hline N17B-C16B & $1.359(5)$ & $\mathrm{C} 19 \mathrm{~B}-\mathrm{H} 192$ & 0.9600 \\
\hline $\mathrm{C} 10 \mathrm{~B}-\mathrm{C} 9 \mathrm{~B}$ & $1.372(6)$ & $\mathrm{C} 19 \mathrm{~B}-\mathrm{H} 193$ & 0.9600 \\
\hline $\mathrm{C} 10 \mathrm{~B}-\mathrm{C} 4 \mathrm{~B}$ & $1.389(5)$ & C9A-H9A & $1.00(4)$ \\
\hline $\mathrm{C} 10 \mathrm{~B}-\mathrm{H} 10 \mathrm{~B}$ & $0.97(4)$ & $\mathrm{C} 14 \mathrm{~B}-\mathrm{C} 18 \mathrm{~B}$ & $1.506(6)$ \\
\hline $\mathrm{C} 7 \mathrm{~A}-\mathrm{N} 8 \mathrm{~A}$ & $1.347(5)$ & $\mathrm{C} 18 \mathrm{~B}-\mathrm{H} 183$ & 0.9600 \\
\hline $\mathrm{C} 7 \mathrm{~A}-\mathrm{C} 6 \mathrm{~A}$ & $1.401(5)$ & $\mathrm{C} 18 \mathrm{~B}-\mathrm{H} 182$ & 0.9600 \\
\hline $\mathrm{C} 7 \mathrm{~A}-\mathrm{C} 9 \mathrm{~A}$ & $1.403(5)$ & $\mathrm{C} 18 \mathrm{~B}-\mathrm{H} 181$ & 0.9600 \\
\hline $\mathrm{C} 4 \mathrm{~A}-\mathrm{C} 10 \mathrm{~A}$ & $1.387(5)$ & $\mathrm{C} 14 \mathrm{~A}-\mathrm{C} 18 \mathrm{~A}$ & $1.505(8)$ \\
\hline $\mathrm{C} 4 \mathrm{~A}-\mathrm{C} 5 \mathrm{~A}$ & $1.390(5)$ & $\mathrm{C} 19 \mathrm{~A}-\mathrm{H} 19 \mathrm{X}$ & 0.9600 \\
\hline $\mathrm{C} 12 \mathrm{~B}-\mathrm{N} 13 \mathrm{~B}$ & $1.325(5)$ & $\mathrm{C} 19 \mathrm{~A}-\mathrm{H} 19 \mathrm{Z}$ & 0.9600 \\
\hline $\mathrm{C} 12 \mathrm{~B}-\mathrm{N} 11 \mathrm{~B}$ & $1.403(5)$ & $\mathrm{C} 19 \mathrm{~A}-\mathrm{H} 19 \mathrm{Y}$ & 0.9600 \\
\hline N11B-H11B & $0.80(4)$ & $\mathrm{C} 18 \mathrm{~A}-\mathrm{H} 18 \mathrm{Z}$ & 0.9600 \\
\hline $\mathrm{C} 10 \mathrm{~A}-\mathrm{C} 9 \mathrm{~A}$ & $1.369(6)$ & $\mathrm{C} 18 \mathrm{~A}-\mathrm{H} 18 \mathrm{X}$ & 0.9600 \\
\hline $\mathrm{C} 10 \mathrm{~A}-\mathrm{H} 10 \mathrm{~A}$ & $0.95(4)$ & $\mathrm{C} 18 \mathrm{~A}-\mathrm{H} 18 \mathrm{Y}$ & 0.9600 \\
\hline
\end{tabular}




\begin{tabular}{|c|c|c|c|}
\hline $\mathrm{N} 17 \mathrm{~A}-\mathrm{C} 12 \mathrm{~A}$ & $1.324(5)$ & $\mathrm{N} 20 \mathrm{~B}-\mathrm{C} 25 \mathrm{~B}$ & $1.306(7)$ \\
\hline $\mathrm{N} 17 \mathrm{~A}-\mathrm{C} 16 \mathrm{~A}$ & $1.354(6)$ & $\mathrm{N} 20 \mathrm{~B}-\mathrm{C} 21 \mathrm{~B}$ & $1.318(6)$ \\
\hline $\mathrm{C} 6 \mathrm{~A}-\mathrm{C} 5 \mathrm{~A}$ & $1.362(6)$ & $\mathrm{C} 21 \mathrm{~B}-\mathrm{C} 22 \mathrm{~B}$ & $1.380(7)$ \\
\hline $\mathrm{C} 6 \mathrm{~A}-\mathrm{H} 6 \mathrm{~A}$ & $0.90(4)$ & $\mathrm{C} 21 \mathrm{~B}-\mathrm{H} 21 \mathrm{~B}$ & 0.9300 \\
\hline $\mathrm{N} 8 \mathrm{~A}-\mathrm{H} 82 \mathrm{~A}$ & $0.78(4)$ & $\mathrm{C} 25 \mathrm{~B}-\mathrm{C} 24 \mathrm{~B}$ & $1.360(8)$ \\
\hline $\mathrm{N} 8 \mathrm{~A}-\mathrm{H} 81 \mathrm{~A}$ & $0.81(5)$ & $\mathrm{C} 25 \mathrm{~B}-\mathrm{H} 25 \mathrm{~B}$ & 0.9300 \\
\hline $\mathrm{N} 13 \mathrm{~B}-\mathrm{C} 14 \mathrm{~B}$ & $1.338(5)$ & $\mathrm{C} 23 \mathrm{~B}-\mathrm{C} 24 \mathrm{~B}$ & $1.340(8)$ \\
\hline $\mathrm{C} 5 \mathrm{~A}-\mathrm{H} 5 \mathrm{~A}$ & $0.96(4)$ & $\mathrm{C} 23 \mathrm{~B}-\mathrm{C} 22 \mathrm{~B}$ & $1.345(8)$ \\
\hline $\mathrm{N} 11 \mathrm{~A}-\mathrm{C} 12 \mathrm{~A}$ & $1.379(5)$ & $\mathrm{C} 23 \mathrm{~B}-\mathrm{H} 23 \mathrm{~B}$ & 0.9300 \\
\hline $\mathrm{N} 11 \mathrm{~A}-\mathrm{H} 11 \mathrm{~A}$ & $0.81(4)$ & $\mathrm{C} 24 \mathrm{~B}-\mathrm{H} 24 \mathrm{~B}$ & 0.9300 \\
\hline $\mathrm{C} 4 \mathrm{~B}-\mathrm{C} 5 \mathrm{~B}$ & $1.383(5)$ & $\mathrm{C} 22 \mathrm{~B}-\mathrm{H} 22 \mathrm{~B}$ & 0.9300 \\
\hline $\mathrm{C} 21 \mathrm{~A}-\mathrm{N} 20 \mathrm{~A}-\mathrm{C} 25 \mathrm{~A}$ & $116.9(5)$ & $\mathrm{C} 14 \mathrm{~B}-\mathrm{C} 15 \mathrm{~B}-\mathrm{H} 15 \mathrm{~B}$ & $124(2)$ \\
\hline $\mathrm{N} 20 \mathrm{~A}-\mathrm{C} 25 \mathrm{~A}-\mathrm{C} 24 \mathrm{~A}$ & $124.8(6)$ & $\mathrm{C} 16 \mathrm{~B}-\mathrm{C} 15 \mathrm{~B}-\mathrm{H} 15 \mathrm{~B}$ & $117(2)$ \\
\hline $\mathrm{N} 20 \mathrm{~A}-\mathrm{C} 25 \mathrm{~A}-\mathrm{H} 25 \mathrm{~A}$ & 117.6 & $\mathrm{C} 6 \mathrm{~B}-\mathrm{C} 5 \mathrm{~B}-\mathrm{C} 4 \mathrm{~B}$ & $119.8(4)$ \\
\hline $\mathrm{C} 24 \mathrm{~A}-\mathrm{C} 25 \mathrm{~A}-\mathrm{H} 25 \mathrm{~A}$ & 117.6 & $\mathrm{C} 6 \mathrm{~B}-\mathrm{C} 5 \mathrm{~B}-\mathrm{H} 5 \mathrm{~B}$ & $119(2)$ \\
\hline $\mathrm{C} 23 \mathrm{~A}-\mathrm{C} 24 \mathrm{~A}-\mathrm{C} 25 \mathrm{~A}$ & $118.0(8)$ & $\mathrm{C} 4 \mathrm{~B}-\mathrm{C} 5 \mathrm{~B}-\mathrm{H} 5 \mathrm{~B}$ & $121(2)$ \\
\hline $\mathrm{C} 23 \mathrm{~A}-\mathrm{C} 24 \mathrm{~A}-\mathrm{H} 24 \mathrm{~A}$ & 121.0 & $\mathrm{~N} 17 \mathrm{~A}-\mathrm{C} 12 \mathrm{~A}-\mathrm{N} 13 \mathrm{~A}$ & $127.3(4)$ \\
\hline $\mathrm{C} 25 \mathrm{~A}-\mathrm{C} 24 \mathrm{~A}-\mathrm{H} 24 \mathrm{~A}$ & 121.0 & $\mathrm{~N} 17 \mathrm{~A}-\mathrm{C} 12 \mathrm{~A}-\mathrm{N} 11 \mathrm{~A}$ & $118.4(4)$ \\
\hline $\mathrm{N} 20 \mathrm{~A}-\mathrm{C} 21 \mathrm{~A}-\mathrm{C} 22 \mathrm{~A}$ & $123.4(7)$ & $\mathrm{N} 13 \mathrm{~A}-\mathrm{C} 12 \mathrm{~A}-\mathrm{N} 11 \mathrm{~A}$ & $114.3(4)$ \\
\hline $\mathrm{N} 20 \mathrm{~A}-\mathrm{C} 21 \mathrm{~A}-\mathrm{H} 21 \mathrm{~A}$ & 118.3 & $\mathrm{C} 10 \mathrm{~B}-\mathrm{C} 9 \mathrm{~B}-\mathrm{C} 7 \mathrm{~B}$ & $121.0(4)$ \\
\hline $\mathrm{C} 22 \mathrm{~A}-\mathrm{C} 21 \mathrm{~A}-\mathrm{H} 21 \mathrm{~A}$ & 118.3 & $\mathrm{C} 10 \mathrm{~B}-\mathrm{C} 9 \mathrm{~B}-\mathrm{H} 9 \mathrm{~B}$ & $123(3)$ \\
\hline $\mathrm{C} 21 \mathrm{~A}-\mathrm{C} 22 \mathrm{~A}-\mathrm{C} 23 \mathrm{~A}$ & $117.8(8)$ & $\mathrm{C} 7 \mathrm{~B}-\mathrm{C} 9 \mathrm{~B}-\mathrm{H} 9 \mathrm{~B}$ & $116(3)$ \\
\hline $\mathrm{C} 21 \mathrm{~A}-\mathrm{C} 22 \mathrm{~A}-\mathrm{H} 22 \mathrm{~A}$ & 121.1 & $\mathrm{C} 7 \mathrm{~B}-\mathrm{N} 8 \mathrm{~B}-\mathrm{H} 81 \mathrm{~B}$ & $114(3)$ \\
\hline $\mathrm{C} 23 \mathrm{~A}-\mathrm{C} 22 \mathrm{~A}-\mathrm{H} 22 \mathrm{~A}$ & 121.1 & $\mathrm{C} 7 \mathrm{~B}-\mathrm{N} 8 \mathrm{~B}-\mathrm{H} 82 \mathrm{~B}$ & $123(5)$ \\
\hline $\mathrm{C} 24 \mathrm{~A}-\mathrm{C} 23 \mathrm{~A}-\mathrm{C} 22 \mathrm{~A}$ & $119.0(7)$ & $\mathrm{H} 81 \mathrm{~B}-\mathrm{N} 8 \mathrm{~B}-\mathrm{H} 82 \mathrm{~B}$ & $122(6)$ \\
\hline $\mathrm{C} 24 \mathrm{~A}-\mathrm{C} 23 \mathrm{~A}-\mathrm{H} 23 \mathrm{~A}$ & 120.5 & $\mathrm{C} 14 \mathrm{~A}-\mathrm{C} 15 \mathrm{~A}-\mathrm{C} 16 \mathrm{~A}$ & $119.6(5)$ \\
\hline $\mathrm{C} 22 \mathrm{~A}-\mathrm{C} 23 \mathrm{~A}-\mathrm{H} 23 \mathrm{~A}$ & 120.5 & $\mathrm{C} 14 \mathrm{~A}-\mathrm{C} 15 \mathrm{~A}-\mathrm{H} 15 \mathrm{~A}$ & $121(4)$ \\
\hline $\mathrm{O} 3 \mathrm{~B}-\mathrm{S} 1 \mathrm{~B}-\mathrm{O} 2 \mathrm{~B}$ & $118.29(17)$ & $\mathrm{C} 16 \mathrm{~A}-\mathrm{C} 15 \mathrm{~A}-\mathrm{H} 15 \mathrm{~A}$ & $120(4)$ \\
\hline $\mathrm{O} 3 \mathrm{~B}-\mathrm{S} 1 \mathrm{~B}-\mathrm{N} 11 \mathrm{~B}$ & $109.62(18)$ & $\mathrm{N} 17 \mathrm{~A}-\mathrm{C} 16 \mathrm{~A}-\mathrm{C} 15 \mathrm{~A}$ & $120.3(5)$ \\
\hline $\mathrm{O} 2 \mathrm{~B}-\mathrm{S} 1 \mathrm{~B}-\mathrm{N} 11 \mathrm{~B}$ & $103.88(18)$ & $\mathrm{N} 17 \mathrm{~A}-\mathrm{C} 16 \mathrm{~A}-\mathrm{C} 19 \mathrm{~A}$ & $116.7(5)$ \\
\hline $\mathrm{O} 3 \mathrm{~B}-\mathrm{S} 1 \mathrm{~B}-\mathrm{C} 4 \mathrm{~B}$ & $107.37(17)$ & $\mathrm{C} 15 \mathrm{~A}-\mathrm{C} 16 \mathrm{~A}-\mathrm{C} 19 \mathrm{~A}$ & $123.0(5)$ \\
\hline $\mathrm{O} 2 \mathrm{~B}-\mathrm{S} 1 \mathrm{~B}-\mathrm{C} 4 \mathrm{~B}$ & $109.47(17)$ & $\mathrm{N} 8 \mathrm{~B}-\mathrm{C} 7 \mathrm{~B}-\mathrm{C} 6 \mathrm{~B}$ & $121.2(5)$ \\
\hline $\mathrm{N} 11 \mathrm{~B}-\mathrm{S} 1 \mathrm{~B}-\mathrm{C} 4 \mathrm{~B}$ & $107.80(18)$ & $\mathrm{N} 8 \mathrm{~B}-\mathrm{C} 7 \mathrm{~B}-\mathrm{C} 9 \mathrm{~B}$ & $121.5(4)$ \\
\hline $\mathrm{O} 3 \mathrm{~A}-\mathrm{S} 1 \mathrm{~A}-\mathrm{O} 2 \mathrm{~A}$ & $118.69(19)$ & $\mathrm{C} 6 \mathrm{~B}-\mathrm{C} 7 \mathrm{~B}-\mathrm{C} 9 \mathrm{~B}$ & $117.3(4)$ \\
\hline $\mathrm{O} 3 \mathrm{~A}-\mathrm{S} 1 \mathrm{~A}-\mathrm{N} 11 \mathrm{~A}$ & $109.4(2)$ & $\mathrm{C} 16 \mathrm{~B}-\mathrm{C} 19 \mathrm{~B}-\mathrm{H} 191$ & 109.5 \\
\hline $\mathrm{O} 2 \mathrm{~A}-\mathrm{S} 1 \mathrm{~A}-\mathrm{N} 11 \mathrm{~A}$ & $103.3(2)$ & $\mathrm{C} 16 \mathrm{~B}-\mathrm{C} 19 \mathrm{~B}-\mathrm{H} 192$ & 109.5 \\
\hline $\mathrm{O} 3 \mathrm{~A}-\mathrm{S} 1 \mathrm{~A}-\mathrm{C} 4 \mathrm{~A}$ & $108.77(18)$ & $\mathrm{H} 191-\mathrm{C} 19 \mathrm{~B}-\mathrm{H} 192$ & 109.5 \\
\hline $\mathrm{O} 2 \mathrm{~A}-\mathrm{S} 1 \mathrm{~A}-\mathrm{C} 4 \mathrm{~A}$ & $108.91(16)$ & $\mathrm{C} 16 \mathrm{~B}-\mathrm{C} 19 \mathrm{~B}-\mathrm{H} 193$ & 109.5 \\
\hline $\mathrm{N} 11 \mathrm{~A}-\mathrm{S} 1 \mathrm{~A}-\mathrm{C} 4 \mathrm{~A}$ & $107.25(19)$ & $\mathrm{H} 191-\mathrm{C} 19 \mathrm{~B}-\mathrm{H} 193$ & 109.5 \\
\hline $\mathrm{C} 12 \mathrm{~B}-\mathrm{N} 17 \mathrm{~B}-\mathrm{C} 16 \mathrm{~B}$ & $115.9(3)$ & $\mathrm{H} 192-\mathrm{C} 19 \mathrm{~B}-\mathrm{H} 193$ & 109.5 \\
\hline $\mathrm{C} 9 \mathrm{~B}-\mathrm{C} 10 \mathrm{~B}-\mathrm{C} 4 \mathrm{~B}$ & $120.1(4)$ & $\mathrm{C} 10 \mathrm{~A}-\mathrm{C} 9 \mathrm{~A}-\mathrm{C} 7 \mathrm{~A}$ & $120.5(4)$ \\
\hline $\mathrm{C} 9 \mathrm{~B}-\mathrm{C} 10 \mathrm{~B}-\mathrm{H} 10 \mathrm{~B}$ & $124(2)$ & $\mathrm{C} 10 \mathrm{~A}-\mathrm{C} 9 \mathrm{~A}-\mathrm{H} 9 \mathrm{~A}$ & $117(2)$ \\
\hline $\mathrm{C} 4 \mathrm{~B}-\mathrm{C} 10 \mathrm{~B}-\mathrm{H} 10 \mathrm{~B}$ & $116(2)$ & $\mathrm{C} 7 \mathrm{~A}-\mathrm{C} 9 \mathrm{~A}-\mathrm{H} 9 \mathrm{~A}$ & $122(2)$ \\
\hline $\mathrm{N} 8 \mathrm{~A}-\mathrm{C} 7 \mathrm{~A}-\mathrm{C} 6 \mathrm{~A}$ & $120.9(4)$ & $\mathrm{N} 13 \mathrm{~B}-\mathrm{C} 14 \mathrm{~B}-\mathrm{C} 15 \mathrm{~B}$ & $121.8(4)$ \\
\hline $\mathrm{N} 8 \mathrm{~A}-\mathrm{C} 7 \mathrm{~A}-\mathrm{C} 9 \mathrm{~A}$ & $121.3(4)$ & $\mathrm{N} 13 \mathrm{~B}-\mathrm{C} 14 \mathrm{~B}-\mathrm{C} 18 \mathrm{~B}$ & $115.8(4)$ \\
\hline $\mathrm{C} 6 \mathrm{~A}-\mathrm{C} 7 \mathrm{~A}-\mathrm{C} 9 \mathrm{~A}$ & $117.8(4)$ & $\mathrm{C} 15 \mathrm{~B}-\mathrm{C} 14 \mathrm{~B}-\mathrm{C} 18 \mathrm{~B}$ & $122.4(4)$ \\
\hline $\mathrm{C} 10 \mathrm{~A}-\mathrm{C} 4 \mathrm{~A}-\mathrm{C} 5 \mathrm{~A}$ & $119.1(4)$ & $\mathrm{C} 14 \mathrm{~B}-\mathrm{C} 18 \mathrm{~B}-\mathrm{H} 183$ & 109.5 \\
\hline
\end{tabular}




\begin{tabular}{|c|c|}
\hline $\mathrm{C} 10 \mathrm{~A}-\mathrm{C} 4 \mathrm{~A}-\mathrm{S} 1 \mathrm{~A}$ & $119.6(3)$ \\
\hline $\mathrm{C} 5 \mathrm{~A}-\mathrm{C} 4 \mathrm{~A}-\mathrm{S} 1 \mathrm{~A}$ & $121.4(3)$ \\
\hline $\mathrm{N} 13 \mathrm{~B}-\mathrm{C} 12 \mathrm{~B}-\mathrm{N} 17 \mathrm{~B}$ & $128.2(4)$ \\
\hline $\mathrm{N} 13 \mathrm{~B}-\mathrm{C} 12 \mathrm{~B}-\mathrm{N} 11 \mathrm{~B}$ & $114.5(4)$ \\
\hline $\mathrm{N} 17 \mathrm{~B}-\mathrm{C} 12 \mathrm{~B}-\mathrm{N} 11 \mathrm{~B}$ & $117.3(3)$ \\
\hline $\mathrm{C} 12 \mathrm{~B}-\mathrm{N} 11 \mathrm{~B}-\mathrm{S} 1 \mathrm{~B}$ & $125.6(3)$ \\
\hline $\mathrm{C} 12 \mathrm{~B}-\mathrm{N} 11 \mathrm{~B}-\mathrm{H} 11 \mathrm{~B}$ & $120(3)$ \\
\hline $\mathrm{S} 1 \mathrm{~B}-\mathrm{N} 11 \mathrm{~B}-\mathrm{H} 11 \mathrm{~B}$ & $107(3)$ \\
\hline $\mathrm{C} 9 \mathrm{~A}-\mathrm{C} 10 \mathrm{~A}-\mathrm{C} 4 \mathrm{~A}$ & $121.0(4)$ \\
\hline $\mathrm{C} 9 \mathrm{~A}-\mathrm{C} 10 \mathrm{~A}-\mathrm{H} 10 \mathrm{~A}$ & $121(2)$ \\
\hline $\mathrm{C} 4 \mathrm{~A}-\mathrm{C} 10 \mathrm{~A}-\mathrm{H} 10 \mathrm{~A}$ & $118(2)$ \\
\hline $\mathrm{C} 12 \mathrm{~A}-\mathrm{N} 17 \mathrm{~A}-\mathrm{C} 16 \mathrm{~A}$ & $116.1(4)$ \\
\hline $\mathrm{C} 5 \mathrm{~A}-\mathrm{C} 6 \mathrm{~A}-\mathrm{C} 7 \mathrm{~A}$ & $121.5(4)$ \\
\hline $\mathrm{C} 5 \mathrm{~A}-\mathrm{C} 6 \mathrm{~A}-\mathrm{H} 6 \mathrm{~A}$ & $122(2)$ \\
\hline $\mathrm{C} 7 \mathrm{~A}-\mathrm{C} 6 \mathrm{~A}-\mathrm{H} 6 \mathrm{~A}$ & $117(2)$ \\
\hline $\mathrm{C} 7 \mathrm{~A}-\mathrm{N} 8 \mathrm{~A}-\mathrm{H} 82 \mathrm{~A}$ & $120(3)$ \\
\hline $\mathrm{C} 7 \mathrm{~A}-\mathrm{N} 8 \mathrm{~A}-\mathrm{H} 81 \mathrm{~A}$ & $117(3)$ \\
\hline $\mathrm{H} 82 \mathrm{~A}-\mathrm{N} 8 \mathrm{~A}-\mathrm{H} 81 \mathrm{~A}$ & $121(5)$ \\
\hline $\mathrm{C} 12 \mathrm{~B}-\mathrm{N} 13 \mathrm{~B}-\mathrm{C} 14 \mathrm{~B}$ & $115.0(4)$ \\
\hline $\mathrm{C} 6 \mathrm{~A}-\mathrm{C} 5 \mathrm{~A}-\mathrm{C} 4 \mathrm{~A}$ & $120.2(4)$ \\
\hline $\mathrm{C} 6 \mathrm{~A}-\mathrm{C} 5 \mathrm{~A}-\mathrm{H} 5 \mathrm{~A}$ & $124(2)$ \\
\hline $\mathrm{C} 4 \mathrm{~A}-\mathrm{C} 5 \mathrm{~A}-\mathrm{H} 5 \mathrm{~A}$ & $116(2)$ \\
\hline $\mathrm{C} 12 \mathrm{~A}-\mathrm{N} 11 \mathrm{~A}-\mathrm{S} 1 \mathrm{~A}$ & $125.2(3)$ \\
\hline $\mathrm{C} 12 \mathrm{~A}-\mathrm{N} 11 \mathrm{~A}-\mathrm{H} 11 \mathrm{~A}$ & $125(4)$ \\
\hline S1A-N11A-H11A & $106(4)$ \\
\hline $\mathrm{C} 5 \mathrm{~B}-\mathrm{C} 4 \mathrm{~B}-\mathrm{C} 10 \mathrm{~B}$ & $119.8(4)$ \\
\hline $\mathrm{C} 5 \mathrm{~B}-\mathrm{C} 4 \mathrm{~B}-\mathrm{S} 1 \mathrm{~B}$ & $120.9(3)$ \\
\hline $\mathrm{C} 10 \mathrm{~B}-\mathrm{C} 4 \mathrm{~B}-\mathrm{S} 1 \mathrm{~B}$ & $119.3(3)$ \\
\hline $\mathrm{C} 5 \mathrm{~B}-\mathrm{C} 6 \mathrm{~B}-\mathrm{C} 7 \mathrm{~B}$ & $122.0(5)$ \\
\hline $\mathrm{C} 5 \mathrm{~B}-\mathrm{C} 6 \mathrm{~B}-\mathrm{H} 6 \mathrm{~B}$ & $123(3)$ \\
\hline $\mathrm{C} 7 \mathrm{~B}-\mathrm{C} 6 \mathrm{~B}-\mathrm{H} 6 \mathrm{~B}$ & $115(3)$ \\
\hline $\mathrm{C} 12 \mathrm{~A}-\mathrm{N} 13 \mathrm{~A}-\mathrm{C} 14 \mathrm{~A}$ & $115.8(4)$ \\
\hline $\mathrm{N} 17 \mathrm{~B}-\mathrm{C} 16 \mathrm{~B}-\mathrm{C} 15 \mathrm{~B}$ & $119.6(4)$ \\
\hline $\mathrm{N} 17 \mathrm{~B}-\mathrm{C} 16 \mathrm{~B}-\mathrm{C} 19 \mathrm{~B}$ & $116.8(4)$ \\
\hline $\mathrm{C} 15 \mathrm{~B}-\mathrm{C} 16 \mathrm{~B}-\mathrm{C} 19 \mathrm{~B}$ & $123.6(4)$ \\
\hline $\mathrm{C} 14 \mathrm{~B}-\mathrm{C} 15 \mathrm{~B}-\mathrm{C} 16 \mathrm{~B}$ & $119.4(4)$ \\
\hline $\mathrm{C} 21 \mathrm{~A}-\mathrm{N} 20 \mathrm{~A}-\mathrm{C} 25 \mathrm{~A}-\mathrm{C} 24 \mathrm{~A}$ & $0.7(9)$ \\
\hline $\mathrm{N} 20 \mathrm{~A}-\mathrm{C} 25 \mathrm{~A}-\mathrm{C} 24 \mathrm{~A}-\mathrm{C} 23 \mathrm{~A}$ & $-1.2(11)$ \\
\hline $\mathrm{C} 25 \mathrm{~A}-\mathrm{N} 20 \mathrm{~A}-\mathrm{C} 21 \mathrm{~A}-\mathrm{C} 22 \mathrm{~A}$ & $-1.5(11)$ \\
\hline $\mathrm{N} 20 \mathrm{~A}-\mathrm{C} 21 \mathrm{~A}-\mathrm{C} 22 \mathrm{~A}-\mathrm{C} 23 \mathrm{~A}$ & $2.9(15)$ \\
\hline $\mathrm{C} 25 \mathrm{~A}-\mathrm{C} 24 \mathrm{~A}-\mathrm{C} 23 \mathrm{~A}-\mathrm{C} 22 \mathrm{~A}$ & $2.6(13)$ \\
\hline $\mathrm{C} 21 \mathrm{~A}-\mathrm{C} 22 \mathrm{~A}-\mathrm{C} 23 \mathrm{~A}-\mathrm{C} 24 \mathrm{~A}$ & $-3.3(15)$ \\
\hline $\mathrm{O} 3 \mathrm{~A}-\mathrm{S} 1 \mathrm{~A}-\mathrm{C} 4 \mathrm{~A}-\mathrm{C} 10 \mathrm{~A}$ & $166.0(3)$ \\
\hline $\mathrm{O} 2 \mathrm{~A}-\mathrm{S} 1 \mathrm{~A}-\mathrm{C} 4 \mathrm{~A}-\mathrm{C} 10 \mathrm{~A}$ & $35.2(4)$ \\
\hline $\mathrm{N} 11 \mathrm{~A}-\mathrm{S} 1 \mathrm{~A}-\mathrm{C} 4 \mathrm{~A}-\mathrm{C} 10 \mathrm{~A}$ & $-75.9(4)$ \\
\hline $\mathrm{O} 3 \mathrm{~A}-\mathrm{S} 1 \mathrm{~A}-\mathrm{C} 4 \mathrm{~A}-\mathrm{C} 5 \mathrm{~A}$ & $-14.6(4)$ \\
\hline $\mathrm{O} 2 \mathrm{~A}-\mathrm{S} 1 \mathrm{~A}-\mathrm{C} 4 \mathrm{~A}-\mathrm{C} 5 \mathrm{~A}$ & $-145.3(3)$ \\
\hline
\end{tabular}

\begin{tabular}{|c|c|}
\hline $\mathrm{C} 14 \mathrm{~B}-\mathrm{C} 18 \mathrm{~B}-\mathrm{H} 182$ & 109.5 \\
\hline $\mathrm{H} 183-\mathrm{C} 18 \mathrm{~B}-\mathrm{H} 182$ & 109.5 \\
\hline $\mathrm{C} 14 \mathrm{~B}-\mathrm{C} 18 \mathrm{~B}-\mathrm{H} 181$ & 109.5 \\
\hline $\mathrm{H} 183-\mathrm{C} 18 \mathrm{~B}-\mathrm{H} 181$ & 109.5 \\
\hline $\mathrm{H} 182-\mathrm{C} 18 \mathrm{~B}-\mathrm{H} 181$ & 109.5 \\
\hline $\mathrm{N} 13 \mathrm{~A}-\mathrm{C} 14 \mathrm{~A}-\mathrm{C} 15 \mathrm{~A}$ & $121.0(5)$ \\
\hline $\mathrm{N} 13 \mathrm{~A}-\mathrm{C} 14 \mathrm{~A}-\mathrm{C} 18 \mathrm{~A}$ & $115.3(6)$ \\
\hline $\mathrm{C} 15 \mathrm{~A}-\mathrm{C} 14 \mathrm{~A}-\mathrm{C} 18 \mathrm{~A}$ & $123.7(6)$ \\
\hline $\mathrm{C} 16 \mathrm{~A}-\mathrm{C} 19 \mathrm{~A}-\mathrm{H} 19 \mathrm{X}$ & 109.5 \\
\hline $\mathrm{C} 16 \mathrm{~A}-\mathrm{C} 19 \mathrm{~A}-\mathrm{H} 19 \mathrm{Z}$ & 109.5 \\
\hline $\mathrm{H} 19 \mathrm{X}-\mathrm{C} 19 \mathrm{~A}-\mathrm{H} 19 \mathrm{Z}$ & 109.5 \\
\hline $\mathrm{C} 16 \mathrm{~A}-\mathrm{C} 19 \mathrm{~A}-\mathrm{H} 19 \mathrm{Y}$ & 109.5 \\
\hline H19X-C19A-H19Y & 109.5 \\
\hline $\mathrm{H} 19 \mathrm{Z}-\mathrm{C} 19 \mathrm{~A}-\mathrm{H} 19 \mathrm{Y}$ & 109.5 \\
\hline $\mathrm{C} 14 \mathrm{~A}-\mathrm{C} 18 \mathrm{~A}-\mathrm{H} 18 \mathrm{Z}$ & 109.5 \\
\hline $\mathrm{C} 14 \mathrm{~A}-\mathrm{C} 18 \mathrm{~A}-\mathrm{H} 18 \mathrm{X}$ & 109.5 \\
\hline $\mathrm{H} 18 \mathrm{Z}-\mathrm{C} 18 \mathrm{~A}-\mathrm{H} 18 \mathrm{X}$ & 109.5 \\
\hline $\mathrm{C} 14 \mathrm{~A}-\mathrm{C} 18 \mathrm{~A}-\mathrm{H} 18 \mathrm{Y}$ & 109.5 \\
\hline $\mathrm{H} 18 \mathrm{Z}-\mathrm{C} 18 \mathrm{~A}-\mathrm{H} 18 \mathrm{Y}$ & 109.5 \\
\hline $\mathrm{H} 18 \mathrm{X}-\mathrm{C} 18 \mathrm{~A}-\mathrm{H} 18 \mathrm{Y}$ & 109.5 \\
\hline $\mathrm{C} 25 \mathrm{~B}-\mathrm{N} 20 \mathrm{~B}-\mathrm{C} 21 \mathrm{~B}$ & $115.2(5)$ \\
\hline $\mathrm{N} 20 \mathrm{~B}-\mathrm{C} 21 \mathrm{~B}-\mathrm{C} 22 \mathrm{~B}$ & $123.6(5)$ \\
\hline $\mathrm{N} 20 \mathrm{~B}-\mathrm{C} 21 \mathrm{~B}-\mathrm{H} 21 \mathrm{~B}$ & 118.2 \\
\hline $\mathrm{C} 22 \mathrm{~B}-\mathrm{C} 21 \mathrm{~B}-\mathrm{H} 21 \mathrm{~B}$ & 118.2 \\
\hline $\mathrm{N} 20 \mathrm{~B}-\mathrm{C} 25 \mathrm{~B}-\mathrm{C} 24 \mathrm{~B}$ & $124.6(6)$ \\
\hline $\mathrm{N} 20 \mathrm{~B}-\mathrm{C} 25 \mathrm{~B}-\mathrm{H} 25 \mathrm{~B}$ & 117.7 \\
\hline $\mathrm{C} 24 \mathrm{~B}-\mathrm{C} 25 \mathrm{~B}-\mathrm{H} 25 \mathrm{~B}$ & 117.7 \\
\hline $\mathrm{C} 24 \mathrm{~B}-\mathrm{C} 23 \mathrm{~B}-\mathrm{C} 22 \mathrm{~B}$ & $117.8(6)$ \\
\hline $\mathrm{C} 24 \mathrm{~B}-\mathrm{C} 23 \mathrm{~B}-\mathrm{H} 23 \mathrm{~B}$ & 121.1 \\
\hline $\mathrm{C} 22 \mathrm{~B}-\mathrm{C} 23 \mathrm{~B}-\mathrm{H} 23 \mathrm{~B}$ & 121.1 \\
\hline $\mathrm{C} 23 \mathrm{~B}-\mathrm{C} 24 \mathrm{~B}-\mathrm{C} 25 \mathrm{~B}$ & $119.6(6)$ \\
\hline $\mathrm{C} 23 \mathrm{~B}-\mathrm{C} 24 \mathrm{~B}-\mathrm{H} 24 \mathrm{~B}$ & 120.2 \\
\hline $\mathrm{C} 25 \mathrm{~B}-\mathrm{C} 24 \mathrm{~B}-\mathrm{H} 24 \mathrm{~B}$ & 120.2 \\
\hline $\mathrm{C} 23 \mathrm{~B}-\mathrm{C} 22 \mathrm{~B}-\mathrm{C} 21 \mathrm{~B}$ & $119.1(5)$ \\
\hline $\mathrm{C} 23 \mathrm{~B}-\mathrm{C} 22 \mathrm{~B}-\mathrm{H} 22 \mathrm{~B}$ & 120.4 \\
\hline $\mathrm{C} 21 \mathrm{~B}-\mathrm{C} 22 \mathrm{~B}-\mathrm{H} 22 \mathrm{~B}$ & 120.4 \\
\hline $\mathrm{C} 12 \mathrm{~B}-\mathrm{N} 17 \mathrm{~B}-\mathrm{C} 16 \mathrm{~B}-\mathrm{C} 15 \mathrm{~B}$ & $2.5(5)$ \\
\hline $\mathrm{C} 12 \mathrm{~B}-\mathrm{N} 17 \mathrm{~B}-\mathrm{C} 16 \mathrm{~B}-\mathrm{C} 19 \mathrm{~B}$ & $-177.1(4)$ \\
\hline $\mathrm{N} 17 \mathrm{~B}-\mathrm{C} 16 \mathrm{~B}-\mathrm{C} 15 \mathrm{~B}-\mathrm{C} 14 \mathrm{~B}$ & $-0.2(6)$ \\
\hline $\mathrm{C} 19 \mathrm{~B}-\mathrm{C} 16 \mathrm{~B}-\mathrm{C} 15 \mathrm{~B}-\mathrm{C} 14 \mathrm{~B}$ & $179.4(4)$ \\
\hline $\mathrm{C} 7 \mathrm{~B}-\mathrm{C} 6 \mathrm{~B}-\mathrm{C} 5 \mathrm{~B}-\mathrm{C} 4 \mathrm{~B}$ & $0.4(6)$ \\
\hline $\mathrm{C} 10 \mathrm{~B}-\mathrm{C} 4 \mathrm{~B}-\mathrm{C} 5 \mathrm{~B}-\mathrm{C} 6 \mathrm{~B}$ & $1.0(6)$ \\
\hline $\mathrm{S} 1 \mathrm{~B}-\mathrm{C} 4 \mathrm{~B}-\mathrm{C} 5 \mathrm{~B}-\mathrm{C} 6 \mathrm{~B}$ & $-176.8(3)$ \\
\hline $\mathrm{C} 16 \mathrm{~A}-\mathrm{N} 17 \mathrm{~A}-\mathrm{C} 12 \mathrm{~A}-\mathrm{N} 13 \mathrm{~A}$ & $0.1(6)$ \\
\hline $\mathrm{C} 16 \mathrm{~A}-\mathrm{N} 17 \mathrm{~A}-\mathrm{C} 12 \mathrm{~A}-\mathrm{N} 11 \mathrm{~A}$ & $179.7(4)$ \\
\hline $\mathrm{C} 14 \mathrm{~A}-\mathrm{N} 13 \mathrm{~A}-\mathrm{C} 12 \mathrm{~A}-\mathrm{N} 17 \mathrm{~A}$ & $-0.8(7)$ \\
\hline $\mathrm{C} 14 \mathrm{~A}-\mathrm{N} 13 \mathrm{~A}-\mathrm{C} 12 \mathrm{~A}-\mathrm{N} 11 \mathrm{~A}$ & $179.6(4)$ \\
\hline
\end{tabular}




\begin{tabular}{|c|c|c|c|}
\hline $\mathrm{N} 11 \mathrm{~A}-\mathrm{S} 1 \mathrm{~A}-\mathrm{C} 4 \mathrm{~A}-\mathrm{C} 5 \mathrm{~A}$ & $103.6(4)$ & $\mathrm{S} 1 \mathrm{~A}-\mathrm{N} 11 \mathrm{~A}-\mathrm{C} 12 \mathrm{~A}-\mathrm{N} 17 \mathrm{~A}$ & $-12.0(6)$ \\
\hline $\mathrm{C} 16 \mathrm{~B}-\mathrm{N} 17 \mathrm{~B}-\mathrm{C} 12 \mathrm{~B}-\mathrm{N} 13 \mathrm{~B}$ & $-3.5(5)$ & $\mathrm{S} 1 \mathrm{~A}-\mathrm{N} 11 \mathrm{~A}-\mathrm{C} 12 \mathrm{~A}-\mathrm{N} 13 \mathrm{~A}$ & $167.6(3)$ \\
\hline $\mathrm{C} 16 \mathrm{~B}-\mathrm{N} 17 \mathrm{~B}-\mathrm{C} 12 \mathrm{~B}-\mathrm{N} 11 \mathrm{~B}$ & $177.5(3)$ & $\mathrm{C} 4 \mathrm{~B}-\mathrm{C} 10 \mathrm{~B}-\mathrm{C} 9 \mathrm{~B}-\mathrm{C} 7 \mathrm{~B}$ & $-0.1(6)$ \\
\hline $\mathrm{N} 13 \mathrm{~B}-\mathrm{C} 12 \mathrm{~B}-\mathrm{N} 11 \mathrm{~B}-\mathrm{S} 1 \mathrm{~B}$ & $170.0(3)$ & $\mathrm{C} 12 \mathrm{~A}-\mathrm{N} 17 \mathrm{~A}-\mathrm{C} 16 \mathrm{~A}-\mathrm{C} 15 \mathrm{~A}$ & $-0.3(6)$ \\
\hline $\mathrm{N} 17 \mathrm{~B}-\mathrm{C} 12 \mathrm{~B}-\mathrm{N} 11 \mathrm{~B}-\mathrm{S} 1 \mathrm{~B}$ & $-10.8(5)$ & $\mathrm{C} 12 \mathrm{~A}-\mathrm{N} 17 \mathrm{~A}-\mathrm{C} 16 \mathrm{~A}-\mathrm{C} 19 \mathrm{~A}$ & $-178.9(4)$ \\
\hline $\mathrm{O} 3 \mathrm{~B}-\mathrm{S} 1 \mathrm{~B}-\mathrm{N} 11 \mathrm{~B}-\mathrm{C} 12 \mathrm{~B}$ & $60.5(4)$ & $\mathrm{C} 14 \mathrm{~A}-\mathrm{C} 15 \mathrm{~A}-\mathrm{C} 16 \mathrm{~A}-\mathrm{N} 17 \mathrm{~A}$ & $1.1(8)$ \\
\hline $\mathrm{O} 2 \mathrm{~B}-\mathrm{S} 1 \mathrm{~B}-\mathrm{N} 11 \mathrm{~B}-\mathrm{C} 12 \mathrm{~B}$ & $-172.2(3)$ & $\mathrm{C} 14 \mathrm{~A}-\mathrm{C} 15 \mathrm{~A}-\mathrm{C} 16 \mathrm{~A}-\mathrm{C} 19 \mathrm{~A}$ & $179.6(5)$ \\
\hline $\mathrm{C} 4 \mathrm{~B}-\mathrm{S} 1 \mathrm{~B}-\mathrm{N} 11 \mathrm{~B}-\mathrm{C} 12 \mathrm{~B}$ & $-56.1(4)$ & $\mathrm{C} 5 \mathrm{~B}-\mathrm{C} 6 \mathrm{~B}-\mathrm{C} 7 \mathrm{~B}-\mathrm{N} 8 \mathrm{~B}$ & $177.9(4)$ \\
\hline $\mathrm{C} 5 \mathrm{~A}-\mathrm{C} 4 \mathrm{~A}-\mathrm{C} 10 \mathrm{~A}-\mathrm{C} 9 \mathrm{~A}$ & $1.6(7)$ & $\mathrm{C} 5 \mathrm{~B}-\mathrm{C} 6 \mathrm{~B}-\mathrm{C} 7 \mathrm{~B}-\mathrm{C} 9 \mathrm{~B}$ & $-1.6(6)$ \\
\hline $\mathrm{S} 1 \mathrm{~A}-\mathrm{C} 4 \mathrm{~A}-\mathrm{C} 10 \mathrm{~A}-\mathrm{C} 9 \mathrm{~A}$ & $-179.0(4)$ & $\mathrm{C} 10 \mathrm{~B}-\mathrm{C} 9 \mathrm{~B}-\mathrm{C} 7 \mathrm{~B}-\mathrm{N} 8 \mathrm{~B}$ & $-178.1(4)$ \\
\hline $\mathrm{N} 8 \mathrm{~A}-\mathrm{C} 7 \mathrm{~A}-\mathrm{C} 6 \mathrm{~A}-\mathrm{C} 5 \mathrm{~A}$ & $-179.5(4)$ & $\mathrm{C} 10 \mathrm{~B}-\mathrm{C} 9 \mathrm{~B}-\mathrm{C} 7 \mathrm{~B}-\mathrm{C} 6 \mathrm{~B}$ & $1.5(6)$ \\
\hline $\mathrm{C} 9 \mathrm{~A}-\mathrm{C} 7 \mathrm{~A}-\mathrm{C} 6 \mathrm{~A}-\mathrm{C} 5 \mathrm{~A}$ & $1.2(6)$ & $\mathrm{C} 4 \mathrm{~A}-\mathrm{C} 10 \mathrm{~A}-\mathrm{C} 9 \mathrm{~A}-\mathrm{C} 7 \mathrm{~A}$ & $-0.9(7)$ \\
\hline $\mathrm{N} 17 \mathrm{~B}-\mathrm{C} 12 \mathrm{~B}-\mathrm{N} 13 \mathrm{~B}-\mathrm{C} 14 \mathrm{~B}$ & $1.6(6)$ & $\mathrm{N} 8 \mathrm{~A}-\mathrm{C} 7 \mathrm{~A}-\mathrm{C} 9 \mathrm{~A}-\mathrm{C} 10 \mathrm{~A}$ & $-179.7(5)$ \\
\hline $\mathrm{N} 11 \mathrm{~B}-\mathrm{C} 12 \mathrm{~B}-\mathrm{N} 13 \mathrm{~B}-\mathrm{C} 14 \mathrm{~B}$ & $-179.4(3)$ & $\mathrm{C} 6 \mathrm{~A}-\mathrm{C} 7 \mathrm{~A}-\mathrm{C} 9 \mathrm{~A}-\mathrm{C} 10 \mathrm{~A}$ & $-0.5(7)$ \\
\hline $\mathrm{C} 7 \mathrm{~A}-\mathrm{C} 6 \mathrm{~A}-\mathrm{C} 5 \mathrm{~A}-\mathrm{C} 4 \mathrm{~A}$ & $-0.6(6)$ & $\mathrm{C} 12 \mathrm{~B}-\mathrm{N} 13 \mathrm{~B}-\mathrm{C} 14 \mathrm{~B}-\mathrm{C} 15 \mathrm{~B}$ & $1.2(6)$ \\
\hline $\mathrm{C} 10 \mathrm{~A}-\mathrm{C} 4 \mathrm{~A}-\mathrm{C} 5 \mathrm{~A}-\mathrm{C} 6 \mathrm{~A}$ & $-0.8(6)$ & $\mathrm{C} 12 \mathrm{~B}-\mathrm{N} 13 \mathrm{~B}-\mathrm{C} 14 \mathrm{~B}-\mathrm{C} 18 \mathrm{~B}$ & $-179.0(4)$ \\
\hline $\mathrm{S} 1 \mathrm{~A}-\mathrm{C} 4 \mathrm{~A}-\mathrm{C} 5 \mathrm{~A}-\mathrm{C} 6 \mathrm{~A}$ & $179.7(3)$ & $\mathrm{C} 16 \mathrm{~B}-\mathrm{C} 15 \mathrm{~B}-\mathrm{C} 14 \mathrm{~B}-\mathrm{N} 13 \mathrm{~B}$ & $-1.8(7)$ \\
\hline $\mathrm{O} 3 \mathrm{~A}-\mathrm{S} 1 \mathrm{~A}-\mathrm{N} 11 \mathrm{~A}-\mathrm{C} 12 \mathrm{~A}$ & $58.8(4)$ & $\mathrm{C} 16 \mathrm{~B}-\mathrm{C} 15 \mathrm{~B}-\mathrm{C} 14 \mathrm{~B}-\mathrm{C} 18 \mathrm{~B}$ & $178.4(5)$ \\
\hline $\mathrm{O} 2 \mathrm{~A}-\mathrm{S} 1 \mathrm{~A}-\mathrm{N} 11 \mathrm{~A}-\mathrm{C} 12 \mathrm{~A}$ & $-173.9(4)$ & $\mathrm{C} 12 \mathrm{~A}-\mathrm{N} 13 \mathrm{~A}-\mathrm{C} 14 \mathrm{~A}-\mathrm{C} 15 \mathrm{~A}$ & $1.5(7)$ \\
\hline $\mathrm{C} 4 \mathrm{~A}-\mathrm{S} 1 \mathrm{~A}-\mathrm{N} 11 \mathrm{~A}-\mathrm{C} 12 \mathrm{~A}$ & $-58.9(4)$ & $\mathrm{C} 12 \mathrm{~A}-\mathrm{N} 13 \mathrm{~A}-\mathrm{C} 14 \mathrm{~A}-\mathrm{C} 18 \mathrm{~A}$ & $179.6(5)$ \\
\hline $\mathrm{C} 9 \mathrm{~B}-\mathrm{C} 10 \mathrm{~B}-\mathrm{C} 4 \mathrm{~B}-\mathrm{C} 5 \mathrm{~B}$ & $-1.2(6)$ & $\mathrm{C} 16 \mathrm{~A}-\mathrm{C} 15 \mathrm{~A}-\mathrm{C} 14 \mathrm{~A}-\mathrm{N} 13 \mathrm{~A}$ & $-1.7(8)$ \\
\hline $\mathrm{C} 9 \mathrm{~B}-\mathrm{C} 10 \mathrm{~B}-\mathrm{C} 4 \mathrm{~B}-\mathrm{S} 1 \mathrm{~B}$ & $176.7(3)$ & $\mathrm{C} 16 \mathrm{~A}-\mathrm{C} 15 \mathrm{~A}-\mathrm{C} 14 \mathrm{~A}-\mathrm{C} 18 \mathrm{~A}$ & $-179.6(5)$ \\
\hline $\mathrm{O} 3 \mathrm{~B}-\mathrm{S} 1 \mathrm{~B}-\mathrm{C} 4 \mathrm{~B}-\mathrm{C} 5 \mathrm{~B}$ & $-18.3(4)$ & $\mathrm{C} 25 \mathrm{~B}-\mathrm{N} 20 \mathrm{~B}-\mathrm{C} 21 \mathrm{~B}-\mathrm{C} 22 \mathrm{~B}$ & $-0.6(8)$ \\
\hline $\mathrm{O} 2 \mathrm{~B}-\mathrm{S} 1 \mathrm{~B}-\mathrm{C} 4 \mathrm{~B}-\mathrm{C} 5 \mathrm{~B}$ & $-147.9(3)$ & $\mathrm{C} 21 \mathrm{~B}-\mathrm{N} 20 \mathrm{~B}-\mathrm{C} 25 \mathrm{~B}-\mathrm{C} 24 \mathrm{~B}$ & $2.2(11)$ \\
\hline $\mathrm{N} 11 \mathrm{~B}-\mathrm{S} 1 \mathrm{~B}-\mathrm{C} 4 \mathrm{~B}-\mathrm{C} 5 \mathrm{~B}$ & $99.7(3)$ & $\mathrm{C} 22 \mathrm{~B}-\mathrm{C} 23 \mathrm{~B}-\mathrm{C} 24 \mathrm{~B}-\mathrm{C} 25 \mathrm{~B}$ & $2.9(13)$ \\
\hline $\mathrm{O} 3 \mathrm{~B}-\mathrm{S} 1 \mathrm{~B}-\mathrm{C} 4 \mathrm{~B}-\mathrm{C} 10 \mathrm{~B}$ & $163.9(3)$ & $\mathrm{N} 20 \mathrm{~B}-\mathrm{C} 25 \mathrm{~B}-\mathrm{C} 24 \mathrm{~B}-\mathrm{C} 23 \mathrm{~B}$ & $-3.4(14)$ \\
\hline $\mathrm{O} 2 \mathrm{~B}-\mathrm{S} 1 \mathrm{~B}-\mathrm{C} 4 \mathrm{~B}-\mathrm{C} 10 \mathrm{~B}$ & $34.3(4)$ & $\mathrm{C} 24 \mathrm{~B}-\mathrm{C} 23 \mathrm{~B}-\mathrm{C} 22 \mathrm{~B}-\mathrm{C} 21 \mathrm{~B}$ & $-1.4(11)$ \\
\hline $\mathrm{N} 11 \mathrm{~B}-\mathrm{S} 1 \mathrm{~B}-\mathrm{C} 4 \mathrm{~B}-\mathrm{C} 10 \mathrm{~B}$ & $-78.1(4)$ & $\mathrm{N} 20 \mathrm{~B}-\mathrm{C} 21 \mathrm{~B}-\mathrm{C} 22 \mathrm{~B}-\mathrm{C} 23 \mathrm{~B}$ & $0.2(9)$ \\
\hline
\end{tabular}

Hydrogen-bond geometry $\left(\AA,{ }^{\circ}\right)$

\begin{tabular}{lllll}
\hline$D-\mathrm{H} \cdots A$ & $D-\mathrm{H}$ & $\mathrm{H} \cdots A$ & $D \cdots A$ & $D-\mathrm{H} \cdots A$ \\
\hline $\mathrm{N} 8 A-\mathrm{H} 81 A \cdots \mathrm{O} 3 B$ & $0.81(5)$ & $2.34(5)$ & $3.176(6)$ & $172(5)$ \\
$\mathrm{N} 8 A-\mathrm{H} 82 A \cdots \mathrm{O} 3 A$ & $0.78(4)$ & $2.49(4)$ & $3.168(6)$ & $139(4)$ \\
$\mathrm{N} 8 B-\mathrm{H} 81 B \cdots \mathrm{O} 3 B^{\mathrm{i}}$ & $0.82(5)$ & $2.59(5)$ & $3.275(6)$ & $142(4)$ \\
$\mathrm{N} 8 B-\mathrm{H} 81 B \cdots \mathrm{N} 17 B^{\mathrm{i}}$ & $0.82(5)$ & $2.55(5)$ & $3.261(6)$ & $145(4)$ \\
$\mathrm{N} 11 A-\mathrm{H} 11 A \cdots \mathrm{N} 20 A$ & $0.81(4)$ & $2.09(5)$ & $2.894(6)$ & $171(5)$ \\
$\mathrm{N} 11 B-\mathrm{H} 11 B \cdots \mathrm{N} 20 B$ & $0.80(4)$ & $2.04(4)$ & $2.834(5)$ & $174(3)$ \\
\hline
\end{tabular}

Symmetry code: (i) $x, y-1, z$.

(2) 4-Amino-N-(4,6-dimethylpyrimidin-2-yl)benzenesulfonamide pyridine monosolvate

Crystal data

$\mathrm{C}_{12} \mathrm{H}_{14} \mathrm{~N}_{4} \mathrm{O}_{2} \mathrm{~S} \cdot \mathrm{C}_{6} \mathrm{H}_{7} \mathrm{~N}$

$M_{r}=371.46$

Orthorhombic, $P 2{ }_{1} 22_{1}$

$a=9.6920(2) \AA$

$b=25.1673(6) \AA$

$$
\begin{aligned}
& c=7.9853(2) \AA \\
& V=1947.79(8) \AA^{3} \\
& Z=4 \\
& F(000)=784 \\
& D_{\mathrm{x}}=1.267 \mathrm{Mg} \mathrm{m}^{-3}
\end{aligned}
$$


Mo $K \alpha$ radiation, $\lambda=0.71073 \AA$

Cell parameters from 6058 reflections

$\theta=2.3-27.5^{\circ}$

$\mu=0.19 \mathrm{~mm}^{-1}$

Data collection

Bruker Kappa APEXII CCD

diffractometer

$\varphi$ and $\omega$ scans

10022 measured reflections

4438 independent reflections

4082 reflections with $I>2 \sigma(I)$

Refinement

Refinement on $F^{2}$

Least-squares matrix: full

$R\left[F^{2}>2 \sigma\left(F^{2}\right)\right]=0.037$

$w R\left(F^{2}\right)=0.102$

$S=1.03$

4438 reflections

270 parameters

0 restraints

Hydrogen site location: mixed
$T=296 \mathrm{~K}$

Needle, colorless

$0.65 \times 0.60 \times 0.35 \mathrm{~mm}$

$R_{\text {int }}=0.022$

$\theta_{\max }=27.5^{\circ}, \theta_{\min }=1.6^{\circ}$

$h=-7 \rightarrow 12$

$k=-32 \rightarrow 28$

$l=-9 \rightarrow 10$

$\mathrm{H}$ atoms treated by a mixture of independent and constrained refinement

$w=1 /\left[\sigma^{2}\left(F_{\mathrm{o}}^{2}\right)+(0.0685 P)^{2}+0.1007 P\right]$ where $P=\left(F_{\mathrm{o}}^{2}+2 F_{\mathrm{c}}^{2}\right) / 3$

$(\Delta / \sigma)_{\max }=0.001$

$\Delta \rho_{\max }=0.23$ e $\AA^{-3}$

$\Delta \rho_{\min }=-0.28$ e $\AA^{-3}$

Absolute structure: Refined as an inversion twin.

Absolute structure parameter: -0.01 (9)

\section{Special details}

Geometry. All esds (except the esd in the dihedral angle between two 1.s. planes) are estimated using the full covariance matrix. The cell esds are taken into account individually in the estimation of esds in distances, angles and torsion angles; correlations between esds in cell parameters are only used when they are defined by crystal symmetry. An approximate (isotropic) treatment of cell esds is used for estimating esds involving l.s. planes.

Refinement. Refined as a 2-component inversion twin

Fractional atomic coordinates and isotropic or equivalent isotropic displacement parameters $\left(\AA^{2}\right)$

\begin{tabular}{lllll}
\hline & $x$ & $y$ & $z$ & $U_{\text {iso }} * / U_{\text {eq }}$ \\
\hline S1 & $0.44885(5)$ & $0.65278(2)$ & $0.74692(7)$ & $0.04252(15)$ \\
O2 & $0.31271(17)$ & $0.63361(8)$ & $0.7099(2)$ & $0.0599(5)$ \\
O3 & $0.4646(2)$ & $0.69307(7)$ & $0.8725(2)$ & $0.0568(5)$ \\
N13 & $0.7158(2)$ & $0.54388(8)$ & $0.8259(3)$ & $0.0539(5)$ \\
C6 & $0.6878(3)$ & $0.73158(9)$ & $0.4223(3)$ & $0.0495(5)$ \\
N11 & $0.5299(2)$ & $0.59874(7)$ & $0.8069(3)$ & $0.0440(4)$ \\
N8 & $0.7199(3)$ & $0.72280(10)$ & $0.1257(3)$ & $0.0609(6)$ \\
C4 & $0.5271(2)$ & $0.67515(8)$ & $0.5639(3)$ & $0.0388(4)$ \\
C5 & $0.6251(3)$ & $0.71551(9)$ & $0.5680(3)$ & $0.0460(5)$ \\
N17 & $0.74845(19)$ & $0.63772(7)$ & $0.8302(2)$ & $0.0436(4)$ \\
C7 & $0.6565(2)$ & $0.70779(8)$ & $0.2691(3)$ & $0.0442(5)$ \\
C12 & $0.6727(2)$ & $0.59385(8)$ & $0.8216(3)$ & $0.0401(4)$ \\
C9 & $0.5570(3)$ & $0.66678(9)$ & $0.2682(3)$ & $0.0496(5)$ \\
C10 & $0.4941(3)$ & $0.65099(9)$ & $0.4133(3)$ & $0.0451(5)$ \\
C16 & $0.8857(2)$ & $0.63022(11)$ & $0.8340(3)$ & $0.0511(5)$ \\
C15 & $0.9413(3)$ & $0.58014(12)$ & $0.8330(4)$ & $0.0642(7)$ \\
C24 & $0.4181(4)$ & $0.40510(12)$ & $0.7755(4)$ & $0.0715(8)$
\end{tabular}




$\begin{array}{lllll}\text { N20 } & 0.4000(2) & 0.49939(9) & 0.7369(4) & 0.0648(6) \\ \text { C21 } & 0.2893(3) & 0.49161(15) & 0.6425(5) & 0.0752(9) \\ \text { H21 } & 0.244679 & 0.520952 & 0.596926 & 0.090^{*} \\ \text { C25 } & 0.4616(3) & 0.45649(11) & 0.8002(4) & 0.0652(7) \\ \text { H25 } & 0.539742 & 0.461726 & 0.865845 & 0.078^{*} \\ \text { C19 } & 0.9738(3) & 0.67906(13) & 0.8382(5) & 0.0723(8) \\ \text { H19A } & 0.975191 & 0.693219 & 0.949842 & 0.108^{*} \\ \text { H19B } & 0.936751 & 0.705135 & 0.762881 & 0.108^{*} \\ \text { H19C } & 1.066083 & 0.670151 & 0.804634 & 0.108^{*} \\ \text { C14 } & 0.8535(3) & 0.53738(11) & 0.8339(5) & 0.0657(8) \\ \text { C22 } & 0.2380(4) & 0.44171(19) & 0.6095(5) & 0.0879(11) \\ \text { H22 } & 0.160184 & 0.437432 & 0.542666 & 0.105^{*} \\ \text { C26 } & 0.4937(6) & 0.35923(15) & 0.8538(8) & 0.125(2) \\ \text { H26A } & 0.449231 & 0.326556 & 0.823323 & 0.187^{*} \\ \text { H26B } & 0.587379 & 0.358838 & 0.814647 & 0.187^{*} \\ \text { H26C } & 0.492866 & 0.363021 & 0.973420 & 0.187^{*} \\ \text { C18 } & 0.9020(4) & 0.48042(14) & 0.8406(9) & 0.1108(17) \\ \text { H18A } & 0.877941 & 0.462790 & 0.738079 & 0.166^{*} \\ \text { H18B } & 0.858499 & 0.462644 & 0.932975 & 0.166^{*} \\ \text { H18C } & 1.000285 & 0.479600 & 0.855061 & 0.166^{*} \\ \text { C23 } & 0.3029(4) & 0.39881(15) & 0.6761(5) & 0.0830(11) \\ \text { H23 } & 0.269328 & 0.364876 & 0.654559 & 0.100^{*} \\ \text { H5 } & 0.648(3) & 0.7310(10) & 0.679(3) & 0.041(6)^{*} \\ \text { H9 } & 0.531(3) & 0.6502(13) & 0.167(4) & 0.066(8)^{*} \\ \text { H6 } & 0.748(3) & 0.7616(13) & 0.425(4) & 0.065(9)^{*} \\ \text { H8B } & 0.692(3) & 0.7089(13) & 0.031(4) & 0.062(9)^{*} \\ \text { H10 } & 0.424(4) & 0.6244(14) & 0.414(4) & 0.068(8)^{*} \\ \text { H8A } & 0.782(3) & 0.7477(12) & 0.132(4) & 0.054(8)^{*} \\ \text { H11 } & 0.481(3) & 0.5667(14) & 0.780(4) & 0.074(9)^{*} \\ \text { H15 } & 1.031(4) & 0.5753(13) & 0.844(4) & 0.074(10)^{*}\end{array}$

Atomic displacement parameters $\left(\AA^{2}\right)$

\begin{tabular}{lllllll}
\hline & $U^{11}$ & $U^{22}$ & $U^{33}$ & $U^{12}$ & $U^{13}$ & $U^{23}$ \\
\hline S1 & $0.0365(3)$ & $0.0450(3)$ & $0.0461(3)$ & $0.0105(2)$ & $0.0038(2)$ & $0.0067(2)$ \\
O2 & $0.0322(8)$ & $0.0758(12)$ & $0.0717(11)$ & $0.0089(8)$ & $0.0035(7)$ & $0.0193(9)$ \\
O3 & $0.0677(12)$ & $0.0560(10)$ & $0.0466(8)$ & $0.0166(9)$ & $0.0066(9)$ & $-0.0011(7)$ \\
N13 & $0.0430(11)$ & $0.0420(9)$ & $0.0767(13)$ & $0.0055(8)$ & $-0.0122(10)$ & $0.0026(10)$ \\
C6 & $0.0558(15)$ & $0.0391(11)$ & $0.0535(12)$ & $-0.0099(11)$ & $-0.0007(11)$ & $-0.0010(10)$ \\
N11 & $0.0339(9)$ & $0.0384(8)$ & $0.0596(10)$ & $0.0031(7)$ & $-0.0027(8)$ & $0.0098(8)$ \\
N8 & $0.0699(16)$ & $0.0616(14)$ & $0.0511(12)$ & $-0.0179(12)$ & $0.0136(11)$ & $-0.0072(10)$ \\
C4 & $0.0402(11)$ & $0.0355(9)$ & $0.0406(9)$ & $0.0042(8)$ & $0.0025(9)$ & $0.0038(8)$ \\
C5 & $0.0596(15)$ & $0.0351(10)$ & $0.0434(10)$ & $-0.0022(9)$ & $-0.0046(11)$ & $-0.0038(9)$ \\
N17 & $0.0376(9)$ & $0.0435(9)$ & $0.0496(10)$ & $0.0009(7)$ & $0.0028(8)$ & $-0.0002(8)$ \\
C7 & $0.0475(11)$ & $0.0371(9)$ & $0.0481(11)$ & $0.0008(8)$ & $0.0022(10)$ & $0.0020(9)$ \\
C12 & $0.0346(10)$ & $0.0416(10)$ & $0.0440(10)$ & $0.0036(8)$ & $-0.0032(9)$ & $0.0048(9)$ \\
C9 & $0.0587(13)$ & $0.0483(11)$ & $0.0417(11)$ & $-0.0094(10)$ & $-0.0021(11)$ & $-0.0067(9)$ \\
C10 & $0.0470(12)$ & $0.0403(10)$ & $0.0480(10)$ & $-0.0077(9)$ & $-0.0042(9)$ & $0.0004(10)$
\end{tabular}




$\begin{array}{lllllll}\text { C16 } & 0.0377(12) & 0.0607(14) & 0.0548(12) & -0.0026(11) & -0.0001(10) & -0.0054(11) \\ \text { C15 } & 0.0355(12) & 0.0691(16) & 0.0880(19) & 0.0097(13) & -0.0086(14) & -0.0101(15) \\ \text { C24 } & 0.081(2) & 0.0565(14) & 0.0769(19) & -0.0125(14) & 0.0183(17) & -0.0003(14) \\ \text { N20 } & 0.0543(12) & 0.0563(12) & 0.0838(16) & -0.0073(10) & -0.0075(12) & 0.0001(13) \\ \text { C21 } & 0.0542(17) & 0.085(2) & 0.087(2) & -0.0046(16) & -0.0100(15) & -0.0019(18) \\ \text { C25 } & 0.0632(17) & 0.0558(14) & 0.0767(17) & -0.0085(13) & -0.0096(14) & 0.0002(13) \\ \text { C19 } & 0.0449(15) & 0.0752(18) & 0.097(2) & -0.0154(13) & 0.0101(15) & -0.0133(18) \\ \text { C14 } & 0.0503(15) & 0.0541(14) & 0.093(2) & 0.0195(12) & -0.0156(15) & -0.0038(15) \\ \text { C22 } & 0.060(2) & 0.116(3) & 0.088(2) & -0.026(2) & -0.0038(17) & -0.024(2) \\ \text { C26 } & 0.174(6) & 0.060(2) & 0.140(4) & 0.007(3) & 0.011(4) & 0.019(3) \\ \text { C18 } & 0.072(2) & 0.0599(18) & 0.201(5) & 0.0315(17) & -0.028(3) & -0.009(3) \\ \text { C23 } & 0.083(2) & 0.076(2) & 0.089(2) & -0.035(2) & 0.025(2) & -0.0230(19) \\ & & & & & \end{array}$

Geometric parameters $\left(\AA,{ }^{\circ}\right)$

\begin{tabular}{|c|c|c|c|}
\hline $\mathrm{S} 1-\mathrm{O} 3$ & $1.4342(18)$ & $\mathrm{C} 16-\mathrm{C} 19$ & $1.497(4)$ \\
\hline $\mathrm{S} 1-\mathrm{O} 2$ & 1.4357 (19) & $\mathrm{C} 15-\mathrm{C} 14$ & $1.372(4)$ \\
\hline $\mathrm{S} 1-\mathrm{N} 11$ & $1.6421(18)$ & C15-H15 & $0.88(4)$ \\
\hline $\mathrm{S} 1-\mathrm{C} 4$ & $1.740(2)$ & $\mathrm{C} 24-\mathrm{C} 25$ & $1.375(4)$ \\
\hline $\mathrm{N} 13-\mathrm{C} 12$ & $1.326(3)$ & $\mathrm{C} 24-\mathrm{C} 23$ & $1.379(6)$ \\
\hline N13-C14 & $1.346(3)$ & $\mathrm{C} 24-\mathrm{C} 26$ & $1.504(6)$ \\
\hline $\mathrm{C} 6-\mathrm{C} 5$ & $1.373(4)$ & $\mathrm{N} 20-\mathrm{C} 21$ & $1.325(4)$ \\
\hline $\mathrm{C} 6-\mathrm{C} 7$ & $1.396(3)$ & $\mathrm{N} 20-\mathrm{C} 25$ & $1.334(4)$ \\
\hline $\mathrm{C} 6-\mathrm{H} 6$ & $0.96(3)$ & $\mathrm{C} 21-\mathrm{C} 22$ & $1.376(5)$ \\
\hline $\mathrm{N} 11-\mathrm{C} 12$ & $1.394(3)$ & $\mathrm{C} 21-\mathrm{H} 21$ & 0.9300 \\
\hline N11-H11 & $0.96(4)$ & $\mathrm{C} 25-\mathrm{H} 25$ & 0.9300 \\
\hline $\mathrm{N} 8-\mathrm{C} 7$ & $1.354(3)$ & C19-H19A & 0.9600 \\
\hline N8-H8B & $0.88(3)$ & C19-H19B & 0.9600 \\
\hline N8-H8A & $0.87(3)$ & $\mathrm{C} 19-\mathrm{H} 19 \mathrm{C}$ & 0.9600 \\
\hline $\mathrm{C} 4-\mathrm{C} 10$ & $1.385(3)$ & $\mathrm{C} 14-\mathrm{C} 18$ & $1.509(4)$ \\
\hline $\mathrm{C} 4-\mathrm{C} 5$ & $1.392(3)$ & $\mathrm{C} 22-\mathrm{C} 23$ & $1.358(6)$ \\
\hline $\mathrm{C} 5-\mathrm{H} 5$ & $0.99(3)$ & $\mathrm{C} 22-\mathrm{H} 22$ & 0.9300 \\
\hline $\mathrm{N} 17-\mathrm{C} 12$ & $1.328(3)$ & $\mathrm{C} 26-\mathrm{H} 26 \mathrm{~A}$ & 0.9600 \\
\hline $\mathrm{N} 17-\mathrm{C} 16$ & $1.344(3)$ & $\mathrm{C} 26-\mathrm{H} 26 \mathrm{~B}$ & 0.9600 \\
\hline $\mathrm{C} 7-\mathrm{C} 9$ & $1.412(3)$ & $\mathrm{C} 26-\mathrm{H} 26 \mathrm{C}$ & 0.9600 \\
\hline $\mathrm{C} 9-\mathrm{C} 10$ & $1.369(3)$ & C18-H18A & 0.9600 \\
\hline C9- $\mathrm{H} 9$ & $0.94(3)$ & C18-H18B & 0.9600 \\
\hline $\mathrm{C} 10-\mathrm{H} 10$ & $0.95(3)$ & $\mathrm{C} 18-\mathrm{H} 18 \mathrm{C}$ & 0.9600 \\
\hline $\mathrm{C} 16-\mathrm{C} 15$ & $1.371(4)$ & $\mathrm{C} 23-\mathrm{H} 23$ & 0.9300 \\
\hline $\mathrm{O} 3-\mathrm{S} 1-\mathrm{O} 2$ & $118.66(12)$ & $\mathrm{C} 16-\mathrm{C} 15-\mathrm{H} 15$ & $121(2)$ \\
\hline $\mathrm{O} 3-\mathrm{S} 1-\mathrm{N} 11$ & $109.31(11)$ & $\mathrm{C} 14-\mathrm{C} 15-\mathrm{H} 15$ & $120(2)$ \\
\hline $\mathrm{O} 2-\mathrm{S} 1-\mathrm{N} 11$ & $102.80(11)$ & $\mathrm{C} 25-\mathrm{C} 24-\mathrm{C} 23$ & $116.1(3)$ \\
\hline $\mathrm{O} 3-\mathrm{S} 1-\mathrm{C} 4$ & $108.19(11)$ & $\mathrm{C} 25-\mathrm{C} 24-\mathrm{C} 26$ & 120.9 (4) \\
\hline $\mathrm{O} 2-\mathrm{S} 1-\mathrm{C} 4$ & $109.64(11)$ & $\mathrm{C} 23-\mathrm{C} 24-\mathrm{C} 26$ & $123.1(3)$ \\
\hline $\mathrm{N} 11-\mathrm{S} 1-\mathrm{C} 4$ & $107.72(10)$ & $\mathrm{C} 21-\mathrm{N} 20-\mathrm{C} 25$ & $117.3(3)$ \\
\hline $\mathrm{C} 12-\mathrm{N} 13-\mathrm{C} 14$ & $115.4(2)$ & $\mathrm{N} 20-\mathrm{C} 21-\mathrm{C} 22$ & $122.4(3)$ \\
\hline $\mathrm{C} 5-\mathrm{C} 6-\mathrm{C} 7$ & $121.3(2)$ & $\mathrm{N} 20-\mathrm{C} 21-\mathrm{H} 21$ & 118.8 \\
\hline
\end{tabular}




\begin{tabular}{|c|c|c|c|}
\hline $\mathrm{C} 5-\mathrm{C} 6-\mathrm{H} 6$ & $119(2)$ & $\mathrm{C} 22-\mathrm{C} 21-\mathrm{H} 21$ & 118.8 \\
\hline $\mathrm{C} 7-\mathrm{C} 6-\mathrm{H} 6$ & $119(2)$ & $\mathrm{N} 20-\mathrm{C} 25-\mathrm{C} 24$ & $124.7(3)$ \\
\hline $\mathrm{C} 12-\mathrm{N} 11-\mathrm{S} 1$ & $124.94(16)$ & $\mathrm{N} 20-\mathrm{C} 25-\mathrm{H} 25$ & 117.6 \\
\hline $\mathrm{C} 12-\mathrm{N} 11-\mathrm{H} 11$ & $116(2)$ & $\mathrm{C} 24-\mathrm{C} 25-\mathrm{H} 25$ & 117.6 \\
\hline $\mathrm{S} 1-\mathrm{N} 11-\mathrm{H} 11$ & $113(2)$ & $\mathrm{C} 16-\mathrm{C} 19-\mathrm{H} 19 \mathrm{~A}$ & 109.5 \\
\hline $\mathrm{C} 7-\mathrm{N} 8-\mathrm{H} 8 \mathrm{~B}$ & $119(2)$ & $\mathrm{C} 16-\mathrm{C} 19-\mathrm{H} 19 \mathrm{~B}$ & 109.5 \\
\hline $\mathrm{C} 7-\mathrm{N} 8-\mathrm{H} 8 \mathrm{~A}$ & $118(2)$ & $\mathrm{H} 19 \mathrm{~A}-\mathrm{C} 19-\mathrm{H} 19 \mathrm{~B}$ & 109.5 \\
\hline $\mathrm{H} 8 \mathrm{~B}-\mathrm{N} 8-\mathrm{H} 8 \mathrm{~A}$ & $123(3)$ & $\mathrm{C} 16-\mathrm{C} 19-\mathrm{H} 19 \mathrm{C}$ & 109.5 \\
\hline $\mathrm{C} 10-\mathrm{C} 4-\mathrm{C} 5$ & $119.9(2)$ & $\mathrm{H} 19 \mathrm{~A}-\mathrm{C} 19-\mathrm{H} 19 \mathrm{C}$ & 109.5 \\
\hline $\mathrm{C} 10-\mathrm{C} 4-\mathrm{S} 1$ & $119.13(17)$ & $\mathrm{H} 19 \mathrm{~B}-\mathrm{C} 19-\mathrm{H} 19 \mathrm{C}$ & 109.5 \\
\hline $\mathrm{C} 5-\mathrm{C} 4-\mathrm{S} 1$ & $120.96(17)$ & $\mathrm{N} 13-\mathrm{C} 14-\mathrm{C} 15$ & $121.3(2)$ \\
\hline $\mathrm{C} 6-\mathrm{C} 5-\mathrm{C} 4$ & $119.8(2)$ & $\mathrm{N} 13-\mathrm{C} 14-\mathrm{C} 18$ & $115.2(3)$ \\
\hline $\mathrm{C} 6-\mathrm{C} 5-\mathrm{H} 5$ & $122.9(15)$ & $\mathrm{C} 15-\mathrm{C} 14-\mathrm{C} 18$ & $123.5(3)$ \\
\hline $\mathrm{C} 4-\mathrm{C} 5-\mathrm{H} 5$ & $117.2(15)$ & $\mathrm{C} 23-\mathrm{C} 22-\mathrm{C} 21$ & $118.9(3)$ \\
\hline $\mathrm{C} 12-\mathrm{N} 17-\mathrm{C} 16$ & $115.6(2)$ & $\mathrm{C} 23-\mathrm{C} 22-\mathrm{H} 22$ & 120.6 \\
\hline $\mathrm{N} 8-\mathrm{C} 7-\mathrm{C} 6$ & $121.5(2)$ & $\mathrm{C} 21-\mathrm{C} 22-\mathrm{H} 22$ & 120.6 \\
\hline $\mathrm{N} 8-\mathrm{C} 7-\mathrm{C} 9$ & $120.6(2)$ & $\mathrm{C} 24-\mathrm{C} 26-\mathrm{H} 26 \mathrm{~A}$ & 109.5 \\
\hline $\mathrm{C} 6-\mathrm{C} 7-\mathrm{C} 9$ & $117.8(2)$ & $\mathrm{C} 24-\mathrm{C} 26-\mathrm{H} 26 \mathrm{~B}$ & 109.5 \\
\hline $\mathrm{N} 13-\mathrm{C} 12-\mathrm{N} 17$ & $127.8(2)$ & $\mathrm{H} 26 \mathrm{~A}-\mathrm{C} 26-\mathrm{H} 26 \mathrm{~B}$ & 109.5 \\
\hline $\mathrm{N} 13-\mathrm{C} 12-\mathrm{N} 11$ & $113.5(2)$ & $\mathrm{C} 24-\mathrm{C} 26-\mathrm{H} 26 \mathrm{C}$ & 109.5 \\
\hline $\mathrm{N} 17-\mathrm{C} 12-\mathrm{N} 11$ & $118.68(19)$ & $\mathrm{H} 26 \mathrm{~A}-\mathrm{C} 26-\mathrm{H} 26 \mathrm{C}$ & 109.5 \\
\hline $\mathrm{C} 10-\mathrm{C} 9-\mathrm{C} 7$ & $120.8(2)$ & $\mathrm{H} 26 \mathrm{~B}-\mathrm{C} 26-\mathrm{H} 26 \mathrm{C}$ & 109.5 \\
\hline $\mathrm{C} 10-\mathrm{C} 9-\mathrm{H} 9$ & $118(2)$ & $\mathrm{C} 14-\mathrm{C} 18-\mathrm{H} 18 \mathrm{~A}$ & 109.5 \\
\hline $\mathrm{C} 7-\mathrm{C} 9-\mathrm{H} 9$ & $121(2)$ & $\mathrm{C} 14-\mathrm{C} 18-\mathrm{H} 18 \mathrm{~B}$ & 109.5 \\
\hline $\mathrm{C} 9-\mathrm{C} 10-\mathrm{C} 4$ & $120.3(2)$ & $\mathrm{H} 18 \mathrm{~A}-\mathrm{C} 18-\mathrm{H} 18 \mathrm{~B}$ & 109.5 \\
\hline $\mathrm{C} 9-\mathrm{C} 10-\mathrm{H} 10$ & $122(2)$ & $\mathrm{C} 14-\mathrm{C} 18-\mathrm{H} 18 \mathrm{C}$ & 109.5 \\
\hline $\mathrm{C} 4-\mathrm{C} 10-\mathrm{H} 10$ & $118(2)$ & $\mathrm{H} 18 \mathrm{~A}-\mathrm{C} 18-\mathrm{H} 18 \mathrm{C}$ & 109.5 \\
\hline $\mathrm{N} 17-\mathrm{C} 16-\mathrm{C} 15$ & $121.2(2)$ & $\mathrm{H} 18 \mathrm{~B}-\mathrm{C} 18-\mathrm{H} 18 \mathrm{C}$ & 109.5 \\
\hline N17-C16-C19 & $116.7(2)$ & $\mathrm{C} 22-\mathrm{C} 23-\mathrm{C} 24$ & $120.6(3)$ \\
\hline $\mathrm{C} 15-\mathrm{C} 16-\mathrm{C} 19$ & $122.0(2)$ & $\mathrm{C} 22-\mathrm{C} 23-\mathrm{H} 23$ & 119.7 \\
\hline $\mathrm{C} 16-\mathrm{C} 15-\mathrm{C} 14$ & $118.5(2)$ & $\mathrm{C} 24-\mathrm{C} 23-\mathrm{H} 23$ & 119.7 \\
\hline $\mathrm{O} 3-\mathrm{S} 1-\mathrm{N} 11-\mathrm{C} 12$ & $-67.8(2)$ & $\mathrm{C} 6-\mathrm{C} 7-\mathrm{C} 9-\mathrm{C} 10$ & $-0.1(4)$ \\
\hline $\mathrm{O} 2-\mathrm{S} 1-\mathrm{N} 11-\mathrm{C} 12$ & $165.2(2)$ & $\mathrm{C} 7-\mathrm{C} 9-\mathrm{C} 10-\mathrm{C} 4$ & $0.0(4)$ \\
\hline $\mathrm{C} 4-\mathrm{S} 1-\mathrm{N} 11-\mathrm{C} 12$ & $49.5(2)$ & $\mathrm{C} 5-\mathrm{C} 4-\mathrm{C} 10-\mathrm{C} 9$ & $-0.3(4)$ \\
\hline $\mathrm{O} 3-\mathrm{S} 1-\mathrm{C} 4-\mathrm{C} 10$ & $-162.20(18)$ & $\mathrm{S} 1-\mathrm{C} 4-\mathrm{C} 10-\mathrm{C} 9$ & $-178.1(2)$ \\
\hline $\mathrm{O} 2-\mathrm{S} 1-\mathrm{C} 4-\mathrm{C} 10$ & $-31.4(2)$ & $\mathrm{C} 12-\mathrm{N} 17-\mathrm{C} 16-\mathrm{C} 15$ & $-1.5(4)$ \\
\hline $\mathrm{N} 11-\mathrm{S} 1-\mathrm{C} 4-\mathrm{C} 10$ & $79.7(2)$ & $\mathrm{C} 12-\mathrm{N} 17-\mathrm{C} 16-\mathrm{C} 19$ & $178.1(2)$ \\
\hline $\mathrm{O} 3-\mathrm{S} 1-\mathrm{C} 4-\mathrm{C} 5$ & $20.0(2)$ & $\mathrm{N} 17-\mathrm{C} 16-\mathrm{C} 15-\mathrm{C} 14$ & $-2.2(4)$ \\
\hline $\mathrm{O} 2-\mathrm{S} 1-\mathrm{C} 4-\mathrm{C} 5$ & $150.80(19)$ & $\mathrm{C} 19-\mathrm{C} 16-\mathrm{C} 15-\mathrm{C} 14$ & $178.2(3)$ \\
\hline $\mathrm{N} 11-\mathrm{S} 1-\mathrm{C} 4-\mathrm{C} 5$ & $-98.0(2)$ & $\mathrm{C} 25-\mathrm{N} 20-\mathrm{C} 21-\mathrm{C} 22$ & $0.1(5)$ \\
\hline $\mathrm{C} 7-\mathrm{C} 6-\mathrm{C} 5-\mathrm{C} 4$ & $-0.7(4)$ & $\mathrm{C} 21-\mathrm{N} 20-\mathrm{C} 25-\mathrm{C} 24$ & $-0.5(5)$ \\
\hline $\mathrm{C} 10-\mathrm{C} 4-\mathrm{C} 5-\mathrm{C} 6$ & $0.6(4)$ & $\mathrm{C} 23-\mathrm{C} 24-\mathrm{C} 25-\mathrm{N} 20$ & $0.7(5)$ \\
\hline $\mathrm{S} 1-\mathrm{C} 4-\mathrm{C} 5-\mathrm{C} 6$ & $178.42(19)$ & $\mathrm{C} 26-\mathrm{C} 24-\mathrm{C} 25-\mathrm{N} 20$ & $-179.3(4)$ \\
\hline $\mathrm{C} 5-\mathrm{C} 6-\mathrm{C} 7-\mathrm{N} 8$ & $-178.7(3)$ & $\mathrm{C} 12-\mathrm{N} 13-\mathrm{C} 14-\mathrm{C} 15$ & $-1.7(5)$ \\
\hline $\mathrm{C} 5-\mathrm{C} 6-\mathrm{C} 7-\mathrm{C} 9$ & $0.5(4)$ & $\mathrm{C} 12-\mathrm{N} 13-\mathrm{C} 14-\mathrm{C} 18$ & $179.4(4)$ \\
\hline $\mathrm{C} 14-\mathrm{N} 13-\mathrm{C} 12-\mathrm{N} 17$ & $-2.6(4)$ & $\mathrm{C} 16-\mathrm{C} 15-\mathrm{C} 14-\mathrm{N} 13$ & $3.9(5)$ \\
\hline $\mathrm{C} 14-\mathrm{N} 13-\mathrm{C} 12-\mathrm{N} 11$ & $177.7(3)$ & $\mathrm{C} 16-\mathrm{C} 15-\mathrm{C} 14-\mathrm{C} 18$ & $-177.2(4)$ \\
\hline
\end{tabular}




$\begin{array}{llll}\mathrm{C} 16-\mathrm{N} 17-\mathrm{C} 12-\mathrm{N} 13 & 4.2(4) & \mathrm{N} 20-\mathrm{C} 21-\mathrm{C} 22-\mathrm{C} 23 & 0.1(6) \\ \mathrm{C} 16-\mathrm{N} 17-\mathrm{C} 12-\mathrm{N} 11 & -176.1(2) & \mathrm{C} 21-\mathrm{C} 22-\mathrm{C} 23-\mathrm{C} 24 & 0.1(6) \\ \mathrm{S} 1-\mathrm{N} 11-\mathrm{C} 12-\mathrm{N} 13 & -162.20(19) & \mathrm{C} 25-\mathrm{C} 24-\mathrm{C} 23-\mathrm{C} 22 & -0.5(5) \\ \mathrm{S} 1-\mathrm{N} 11-\mathrm{C} 12-\mathrm{N} 17 & 18.1(3) & \mathrm{C} 26-\mathrm{C} 24-\mathrm{C} 23-\mathrm{C} 22 & 179.5(4) \\ \mathrm{N} 8-\mathrm{C} 7-\mathrm{C} 9-\mathrm{C} 10 & 179.1(3) & & \end{array}$

Hydrogen-bond geometry $\left(A,{ }^{\circ}\right)$

\begin{tabular}{lllll}
\hline$D-\mathrm{H} \cdots A$ & $D-\mathrm{H}$ & $\mathrm{H} \cdots A$ & $D \cdots A$ & $D-\mathrm{H} \cdots A$ \\
\hline $\mathrm{N} 8-\mathrm{H} 8 A^{\cdots} \cdots 3^{\mathrm{i}}$ & 0.87 & 2.31 & $3.1792(3)$ & 173 \\
$\mathrm{~N} 8-\mathrm{H} 8 B \cdots \mathrm{O} 3^{\mathrm{ii}}$ & 0.88 & 2.57 & $3.2821(3)$ & 139 \\
$\mathrm{~N} 8-\mathrm{H} 8 B^{\cdots} \mathrm{N} 17^{\mathrm{ii}}$ & 0.88 & 2.46 & $3.1984(3)$ & 141 \\
$\mathrm{~N} 11-\mathrm{H} 11 \cdots \mathrm{N} 20$ & 0.96 & 1.90 & $2.8550(3)$ & 174 \\
\hline
\end{tabular}

Symmetry codes: (i) $x+1 / 2,-y+1 / 2,-z+1$; (ii) $x, y, z-1$. 